\title{
Multistage quadrature sigma-delta modulators for reconfigurable multi-band analog-digital interface in cognitive radio devices
}

\author{
Jaakko Marttila*, Markus Allén and Mikko Valkama
}

\begin{abstract}
This article addresses the design, analysis, and parameterization of reconfigurable multi-band noise and signal transfer functions (NTF and STF), realized with multistage quadrature $\Sigma \Delta$ modulator (Q $\Sigma \Delta \mathrm{M}$ ) concept and complex-valued in-phase/quadrature (I/Q) signal processing. Such multi-band scheme was already proposed earlier by the authors at a preliminary level, and is here developed further toward flexible and reconfigurable A/D interface for cognitive radio (CR) receivers enabling efficient parallel reception of multiple noncontiguous frequency slices. Owing to straightforward parameterization, the NTF and the STF of the multistage $\mathrm{Q} \Sigma \Delta \mathrm{M}$ can be adapted to input signal conditions based on spectrum-sensing information. It is also shown in the article through closed-form response analysis that the so-called mirror-frequency-rejecting STF design can offer additional operating robustness in challenging scenarios, such as the presence of strong mirror-frequency blocking signals under I/Q imbalance, which is an unavoidable practical problem with quadrature circuits. The mirror-frequency interference stemming from these blockers is analyzed with a novel analytic closed-form I/Q imbalance model for multistage $\mathrm{Q} \Sigma \Delta$ Ms with arbitrary number of stages. Concrete examples are given with three-stage $\mathrm{Q} \Sigma \Delta \mathrm{M}$, which gives valuable degrees of freedom for the transfer function design. High-order frequency asymmetric multi-band noise shaping is, in general, a valuable asset in CR context offering flexible and frequency agile adaptation capability to differing waveforms to be received and detected. As demonstrated by this article, multistage $\mathrm{Q} \Sigma \Delta \mathrm{Ms}$ can indeed offer these properties together with robust operation without risking stability of the modulator.
\end{abstract}

\section{Introduction}

Nowadays, a growing number of parallel wireless communication standards, together with ever-increasing traffic amounts, create a widely acknowledged need for novel radio solutions, such as emerging cognitive radio $(\mathrm{CR})$ paradigm $[1,2]$. On the other hand, transceiver implementations, especially in mobile terminals, should be smallsized, power efficient, highly integrable, and cheap [3-7]. Thus, it would be valuable to avoid implementing parallel transceiver units for separate communication modes. However, operating band of this kind of software defined radio (SDR) should be extremely wide (even $\mathrm{GHz}$ range),

\section{"Correspondence: jaakko.marttila@tut.fi}

Department of Communications Engineering, Tampere University of Technology, P.O. Box 553, Tampere 33101, Finland and dynamic range of the receiver should be high (several tens of dBs) [5-10]. In addition, the transceiver should be able to adapt to numerous different transmission schemes and waveforms $[4-8,10]$. The SDR concept is considered as a physical layer foundation for CR [1], but these demands create a big challenge for transceiver design, especially for mobile devices.

Particularly, the analog-to-digital (A/D) interface has been identified as a key performance-limiting bottleneck $[1,3,4,8,10-12]$. For example, GSM reception demands high dynamic range, and WLAN and LTE bandwidths, in turn, can be up to $20 \mathrm{MHz}$. Combining this kind of differing radio characteristics set massive demands for the A/D converter (ADC) in the receiver. Traditional Nyquist ADCs (possibly with oversampling) divide the conversion resolution equally on all the frequencies, and thus, if 14-bit 
resolution is needed for one of the signals converted, then similar resolution is used over the whole band even if it would not be necessary [12]. At the same time, in wideband SDR receiver, the resolution demand might be even higher because of the increased dynamic range due to multiple waveforms with differing power levels entering the ADC. On the other hand, $\Sigma \Delta$ ADCs have inherent tradeoff between the sampling frequency and resolution [13]. With narrowband signals (such as GSM), e.g., 14-bit resolution can be achieved with 1-bit quantization because of high oversampling and digital filtering. At the same time, modulator structure can be reconfigured for reception of wideband waveforms to meet differing requirements set by, for example, WLAN or LTE standards $[8,14,15]$.

Based on this, one promising solution for the receiver design in this kind of scenario is wideband direct-conversion or low-IF architecture [16] with a bandpass $\Sigma \Delta$ ADC [8, 14]. Additional degrees of freedom can be obtained by introducing quadrature $\Sigma \Delta$ modulator $(\mathrm{Q} \Sigma \Delta \mathrm{M})$ in the receiver, allowing efficient frequency asymmetric quantization noise shaping $[17,18]$. Furthermore, a multi-band modulator aimed to CR receivers is preliminary proposed in [19] and illustrated with receiver block diagram and principal spectra in Figure 1. This kind of multi-band design for $\mathrm{Q} \Sigma \Delta \mathrm{M}$ offers frequency agile flexibility and reconfigurability based on spectrum-sensing information [20] together with capability of receiving multiple parallel frequency bands [19], which are considered essential when realizing A/D interface for CR solutions [1]. In practice, multiple noise-shaping notches can be created on independent, noncontiguous signal bands. In addition, the center frequencies of these noise notches can be tuned based on the spectrum-sensing information obtained in the receiver.

Noise-shaping capabilities of a single-stage $\mathrm{Q} \Sigma \Delta \mathrm{M}$ are limited by the order of the modulator [18]. However, the order of the overall noise transfer function (NTF) can be increased using cascaded multistage modulator [21-23]. Therein, the overall noise shaping is of the combined order of the stages. In a multistage $\mathrm{Q} \Sigma \Delta \mathrm{M}$, the noise notches of the stages can be placed independently, thus further increasing the flexibility of the ADC [21].

Unfortunately, implementing quadrature circuits brings always a challenge of matching the in-phase (I) and quadrature $(\mathrm{Q})$ rails, which should ideally have symmetric component values. Inaccuracies in circuit implementation always shift the designed values, creating imbalance between the rails, known as I/Q imbalance $[18,24]$. This mismatch induces image response of the input signal in addition to the original input, causing mirror-frequency interference (MFI) $[18,24]$. This image response can be modeled mathematically with altered complex conjugate of the signal component. In $\mathrm{Q} \Sigma \Delta \mathrm{Ms}$ generally, the mismatches generate conjugate response for both the input signal and the quantization error $[18,19,25]$, which is a clear difference to mirror-frequency problematics in more traditional receivers. Specifically, feedback branch mismatches have been highlighted as the most important MFI source [23, 26]. From the noise point of view, placing a NTF notch also on mirror frequency to cancel MFI was initially proposed in [18] and discussed further in [27]. This, however, wastes noise shaping performance from the preferred signal point of view and restricts design freedom, especially in multiband scenario. In addition, this does not take the mirroring of the input signal into account. In wideband SDR quadrature receiver, the MFI stemming from the input of the receiver is a crucial viewpoint because of possible blocking signals. Furthermore, alterations to analog circuitry have been proposed in $[26,28,29]$ to minimize the interference. Sharing the components between the branches, however, degrades sampling properties of the modulator [28,

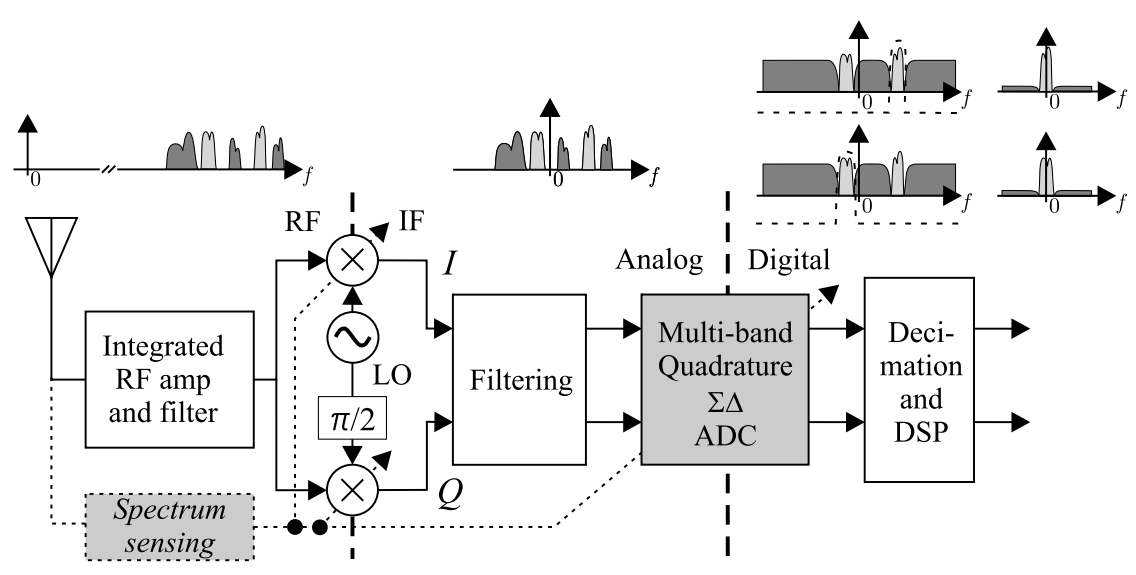

Figure 1 Block diagram of multi-band low-IF quadrature receiver, based on $\mathbf{Q} \boldsymbol{\Sigma} \mathbf{\Delta M}$. Principal spectra, where the two light gray signals are the preferred ones, are illustrating the signal compositions at each stage. 
29]. On the other hand, additional components add to the circuit area and power dissipation of the modulator [26]. In [19], the authors found that mirror-frequency-rejecting signal transfer function (STF) design mitigates the input signal-originating MFI in case of mismatch in the feedback branch of a first-order $\mathrm{Q} \Sigma \Delta \mathrm{M}$. In [21], this idea is extended to cover multi-band design of [19] with a simple two-stage Q $\Sigma \Delta \mathrm{M}$. The feedback I/Q imbalance effects and related digital calibration in two-stage $\mathrm{Q} \Sigma \Delta \mathrm{M}$ are addressed also in [23], where only a frequency-flat STF is considered. In addition, the mirror-frequency-rejecting STF design has a benefit of not demanding additional components to the original $\mathrm{Q} \Sigma \Delta \mathrm{M}$ structure.

In this article, an analytic closed-form model for $\mathrm{Q} \Sigma \Delta \mathrm{M}$ $\mathrm{I} / \mathrm{Q}$ imbalance effects is derived covering multistage modulators with arbitrary number of stages, extending the preliminary analysis with two first-order stages in [21]. Herein, the I/Q imbalance model for second-order $\mathrm{Q} \Sigma \Delta \mathrm{M}$ presented by the authors in [30] is used for each of the stages. Furthermore, design of the transfer functions (STF and NTF) of the stages in such multistage $Q \Sigma \Delta M$ is addressed in detail with emphasis on robust operation under I/Q mismatches. In [31, 32], Q $\Sigma \Delta \mathrm{M}$ STF designs are proposed for reducing the dynamics of the receiver and to filter adjacent channel signals for lowpass and quadrature bandpass modulators, respectively. However, adapting the STF based on spectrum-sensing information is not covered in case of the $\mathrm{Q} \Sigma \Delta \mathrm{M}$ in [31]. In addition, NTF adaptation to frequency handoffs or multi-band reception is not considered in either [31] or [32]. Herein, frequency agile design of the STF and the NTF of an I/Q mismatched multistage $\mathrm{Q} \Sigma \Delta \mathrm{M}$ is discussed taking both the input signal and the quantization noise-oriented MFI into account during multi-band reception.

The push for development of multi-channel ADCs for SDR and CR solutions has been acknowledged, e.g., in [11]. A multi-channel system with parallel ADCs is one possible solution which, however, sets additional burden for size, cost, and power dissipation of the receiver implementation $[11,13]$. On the other hand, quadrature $\Sigma \Delta$ noise shaping makes exploitation of whole quantization precision on the preferred signal bands possible. Three-stage lowpass $\Sigma \Delta$ modulators have traditionally been used only for applications demanding very high resolution [33], but like shown in this article, the $\mathrm{Q} \Sigma \Delta \mathrm{M}$ variant allows noncontiguous placement of the NTF zeros, and thus the quantization precision can be divided on multiple parallel frequency bands. A reconfigurable three-stage converter using lowpass $\Sigma \Delta$ stages together with a pipeline ADC is proposed in [15] for mobile terminals. In comparison, a three-stage $\mathrm{Q} \Sigma \Delta \mathrm{M}$ discussed in this article offers more efficient noise shaping and additional degrees of freedom for the receiver design. These are essential characteristics when heading toward a frequency agile-reconfigurable
$\mathrm{ADC}$ for $\mathrm{CR}$ receivers. Thus, a multistage $\mathrm{Q} \Sigma \Delta \mathrm{M}$ offers a competent platform for realizing flexible multi-band A/D conversion in $\mathrm{CR}$ devices.

The rest of the article is organized as follows. In Section 2, basics of quadrature $\Sigma \Delta$ modulation are reviewed, while Section 3 presents a closed-form model for I/Q imbalance effects in a second-order $\mathrm{Q} \Sigma \Delta \mathrm{M}$ as a single stage of a multistage modulator and proposes a novel extension of the given model for multistage modulators with arbitrary number of stages. Parameterization and design of the modulator transfer functions in CR receivers in the presence of I/Q mismatches are discussed in Section 4. The receiver system level targets and $\mathrm{Q} \Sigma \Delta \mathrm{M}$ performance are discussed in Section 5. Thereafter, Section 6 presents the results of the designs in the previous section with closedform transfer function analysis and computer simulations. Finally, Section 7 concludes the article.

Short note on terminology and notations: term "order" refers in this article to the order of polynomial(s) in $z$ domain transfer functions, while term "stage" refers to individual $\mathrm{Q} \Sigma \Delta \mathrm{M}$ block in a multistage converter where multiple $\mathrm{Q} \Sigma \Delta \mathrm{M}$ blocks are interconnected. The $z$-domain representations of sequences $x(k)$ and $x^{*}(k)$ are denoted as $X[z]$ and $X^{*}\left[z^{*}\right]$, respectively, where superscript $(\cdot)^{*}$ denotes complex conjugation.

\section{Basics of quadrature $\Sigma \Delta$ modulation}

Quadrature variant of the $\Sigma \Delta$ modulator was originally presented in [18]. The concept is based on the modulator structure similar to the one used in real lowpass and bandpass modulators, but employing complex-valued input and output signals together with complex loop filters (integrators). This complex I/Q signal processing gives additional degree of freedom to response design, allowing for frequency-asymmetric STF and NTF. For analysis purposes, a linear model of the modulator is typically used. In other words, this means that quantization error is assumed to be additive and having no correlation with the input signal. Although not being exactly true, this allows analytic derivation of the transfer functions and has thus been applied widely, e.g., in $[18,33]$. Now, the output of a single-stage $\mathrm{Q} \Sigma \Delta \mathrm{M}$, depicted in Figure 2, is defined as

$$
V^{\text {ideal }}[z]=\operatorname{STF}[z] U[z]+\operatorname{NTF}[z] E[z],
$$

where $\operatorname{STF}[z]$ and $\operatorname{NTF}[z]$ are generally complex-valued functions, and $U[z]$ and $E[z]$ denote $z$-transforms of the input signal and quantization noise, respectively.

The achievable NTF shaping and STF selectivity are defined by the order of the modulator. With Pth-order modulator, it is possible to place $P$ zeros and poles in both transfer functions. This is confirmed by derivation of the transfer functions for the structure presented in Figure 2. The 


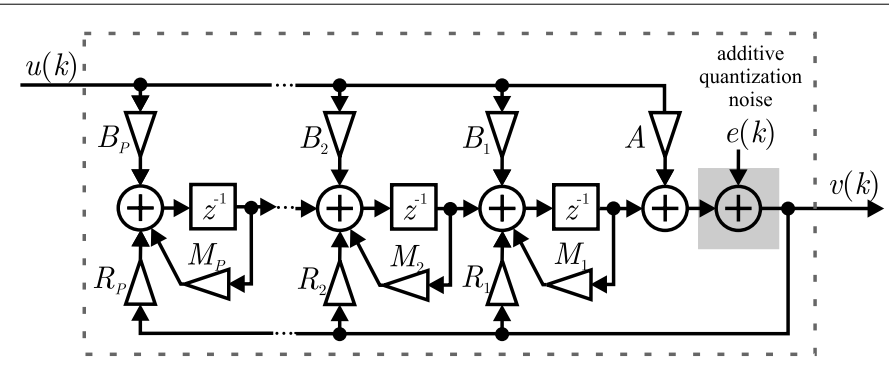

Figure 2 Discrete-time linearized model of a Pth-order $Q \Sigma \Delta M$ with complex-valued signals and coefficients.

NTF of the Pth-order $\mathrm{Q} \Sigma \Delta \mathrm{M}$ is given by

$$
\mathrm{NTF}[z]=\frac{1}{1-\sum_{p=1}^{P} R_{p} \prod_{i=1}^{p} \frac{1}{z-M_{i}}}
$$

and, on the other hand, the corresponding STF is

$$
\operatorname{STF}[z]=\frac{A+\sum_{p=1}^{P} B_{p} \prod_{i=1}^{p} \frac{1}{z-M_{i}}}{1-\sum_{p=1}^{P} R_{p} \prod_{i=1}^{p} \frac{1}{z-M_{i}}}
$$

where $1 /\left(z-M_{i}\right)$ terms are the transfer functions of the complex loop filters (integrators). Both transfer functions have common denominator and thus common poles. It can also be seen that in addition to the loop filters, only the feedback coefficients $R_{p}$ (feeding the output to the loop filters) affect the noise shaping. Thus, input coefficients $A$ (feeding the input to the quantizer) and $B_{p}$ (feeding the input to the loop filters) can be used to tune the STF zeros independent of the NTF.

The NTF zeros are usually placed on the preferred signal band(s) to create the noise-shaping effect. At the same time, the STF zeros can be used to attenuate out-of-band frequencies and thus include some of the receiver selectivity in the $\mathrm{Q} \Sigma \Delta \mathrm{M}$. The transfer function design for $\mathrm{CR}$ is discussed in more detail in Section 4. In the following subsections, multi-band and multistage principles will be presented. These are important concepts, considering reconfigurability in the A/D interface and frequency agile conversion with high-enough resolution in CR devices.

\subsection{Multi-band quadrature $\Sigma \Delta$ ADC for $C R$}

With $\mathrm{Q} \Sigma \Delta \mathrm{M}$ of higher than first order, it is possible to place multiple NTF zeros on the conversion band [18]. Traditional way of exploiting this property has been making the noise-shaping notch wider, thus improving the resolution of the interesting information signal over wider bandwidths [18]. However, in CR-based systems, it is desirable to be able to receive more than one detached frequency bands - and signals - in parallel [1]. The multi-band scheme offers transmission robustness, e.g., in case of appearance of a primary user when the CR user has to vacant that frequency band [1]. In that case, the transmissions can be continued on the other band(s) in use. In addition, if the CR traffic is divided on multiple bands, then lower power levels can be used, and thus the interference generated for primary users is decreased [1].

Multi-band noise shaping without restriction to frequency symmetry is able to respond to this need with noncontiguous NTF notches. This reception scheme is illustrated graphically in Figure 3. The possible number of

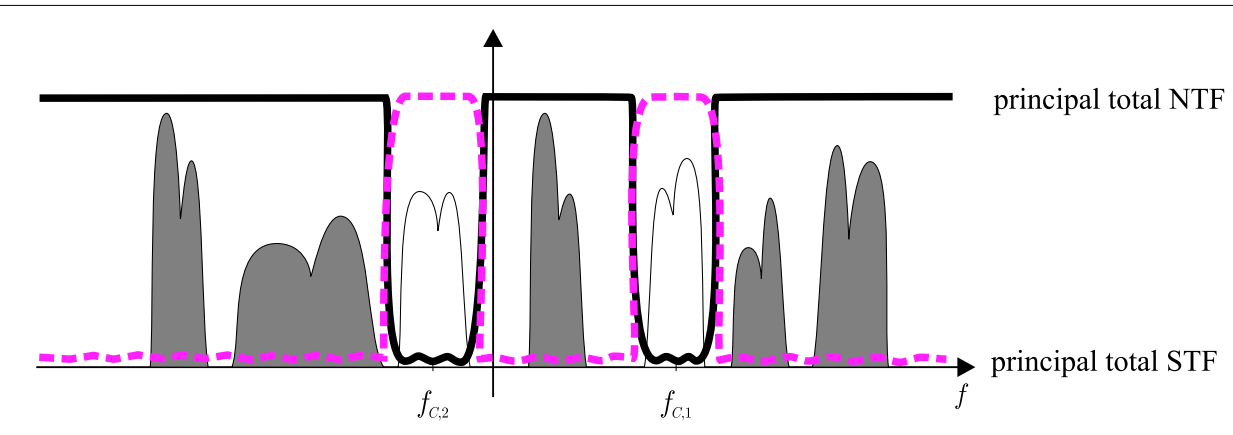

Figure 3 Principal illustration of complex multi-band $\mathbf{Q} \mathbf{\Sigma} \mathbf{M}$ scheme for cognitive radio devices. The light gray signals are assumed to be the preferred ones and principal total STF and NTF are illustrated with magenta dotted and black solid lines, respectively. Quantization noise is shaped away from preferred frequency bands and out-of-band signals are attenuated. 


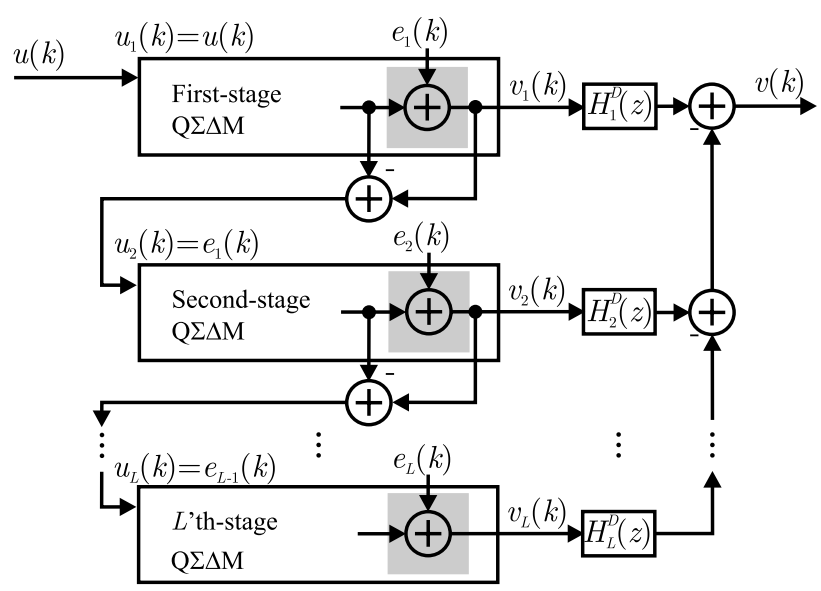

Figure 4 Multistage $\mathbf{Q} \mathbf{\Sigma} \mathbf{\Delta} \mathbf{M}$ with arbitrary-order noise shaping in all the individual stages. Filters $H_{1}^{\mathrm{D}}[z]$ to $H_{L}^{\mathrm{D}}[z]$ are implemented digitally.

these notches is defined by the overall order of the modulator. With multistage $\mathrm{Q} \Sigma \Delta \mathrm{M}$ this is the combined order of all the stages. In addition, the frequencies of the notches can be tuned straightforwardly, e.g., in case of frequency handoff. This tunability of the transfer functions allows also for adaptation to differing waveforms, center frequencies and bandwidths to be received. The resolution and bandwidth demands of the waveforms at hand can be taken into account and the response of the $\mathrm{Q} \Sigma \Delta \mathrm{M}$ can be optimized for the scenario of the moment based on the spectrum-sensing information. Further details on design and parameterization of multi-band transfer functions are given in Section 4.

\section{Multistage quadrature $\Sigma \Delta$ ADC}

Multistage $\Sigma \Delta$ modulators have been introduced to improve resolution, e.g., in case of wideband information signal, when attainable oversampling is limited. This principle was first proposed with lowpass modulator [33], but has thereafter been extended to quadrature bandpass modulator [23, 26]. The block diagram of $L$-stage quadrature $\Sigma \Delta \mathrm{ADC}$ is given in Figure 4 , where all the stages are of arbitrary order. The inputs $u_{l}(k)$ of the $L$ individual stages $(1 \leq l \leq L, l \in \mathbb{Z})$ are defined in the following manner. The input of the first-stage $(l=1)$ is the overall input of the whole structure, i.e., $u_{1}(k)=u(k)$, and for the latter stages, the (ideal) input is the quantization error of the previous stage; thus, $u_{l}(k)=e_{l-1}(k)$ when $2 \leq l \leq L$.

The main goal in multistage $\mathrm{Q} \Sigma \Delta \mathrm{M}$ is to digitize quantization error of the previous stage with the next stage and thereafter subtract it from the output of that previous stage. Owing to the noise shaping in the stages, the digitized error estimate must be filtered in the same way, in order to achieve effective cancelation. Similarly, the output of the first stage must be filtered with digital equivalent of the second-stage STF (e.g., to match the delays). These filters are depicted in Figure 4 with $H_{1}^{\mathrm{D}}[z]$ to $H_{L}^{\mathrm{D}}[z]$. Now, assuming ideal implementation, the final output becomes

$$
V^{\text {ideal }}[z]=\sum_{l=1}^{L}(-1)^{l+1} H_{l}^{\mathrm{D}}[z] V_{l}^{\text {ideal }}[z]
$$

where

$$
\begin{aligned}
& V_{l}^{\text {ideal }}[z]=\operatorname{STF}_{l}^{\text {ideal }}[z] U_{l}[z]+\operatorname{NTF}_{l}^{\text {ideal }}[z] E_{l}[z], \\
& 1 \leq l \leq L, l \in \mathbb{Z}
\end{aligned}
$$

and

$$
H_{l}^{\mathrm{D}}[z]=\frac{H_{1}^{\mathrm{D}}[z] \prod_{l=1}^{L-1} \mathrm{NTF}_{l}^{\text {ideal }}[z]}{\prod_{l=2}^{L} \mathrm{STF}_{l}^{\text {ideal }}[z]}, \quad 1 \leq l \leq L, l \in \mathbb{Z},
$$

to match the analog transfer functions and the digital filters. It is usually chosen that $H_{1}^{\mathrm{D}}[z]=\mathrm{STF}_{2}[z]$, thus giving $H_{2}^{\mathrm{D}}[z]=\mathrm{NTF}_{1}[z]$ and $H_{3}^{\mathrm{D}}[z]=\mathrm{NTF}_{1}[z] \mathrm{NTF}_{2}[z] / \operatorname{STF}_{3}[z]$, etc. With these selections, the quantization errors of the earlier stages are canceled (assuming ideal circuitry), and the overall output of the $L$-stage Q $\Sigma \Delta \mathrm{M}$ becomes $(L \geq 2)$

$$
\begin{aligned}
V^{\text {ideal }}[z]= & \operatorname{STF}_{1}^{\text {ideal }}[z] \operatorname{STF}_{2}^{\mathrm{D}}[z] U[z] \\
& +\frac{\prod_{l=1}^{L} \mathrm{NTF}_{l}^{\text {ideal }}[z]}{\prod_{l=3}^{L} \operatorname{STF}_{l}^{\text {ideal }}[z]} E_{L}[z] \\
= & \operatorname{STF}_{\mathrm{TOT}}^{\text {ideal }}[z] U[z]+\operatorname{NTF}_{\mathrm{TOT}}^{\text {ideal }}[z] E_{L}[z],
\end{aligned}
$$

where only the quantization error of the last stage is present. It is observed that, if three or more stages are used, then special care should be taken in designing the STF of 


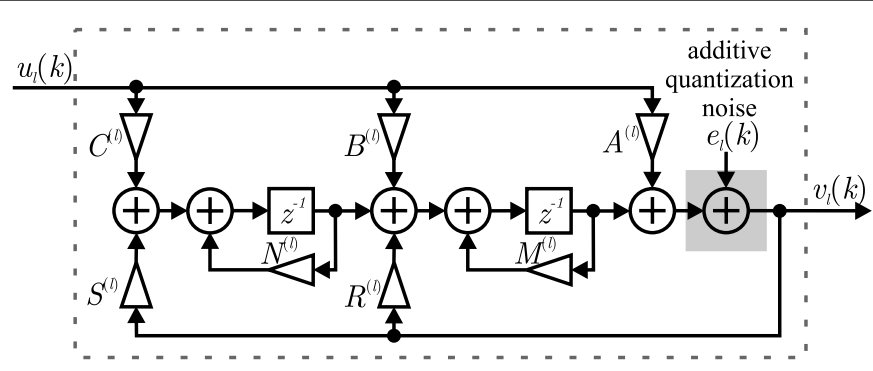

Figure 5 Discrete-time-linearized model of the Ith second-order $Q \Sigma \Delta M$ stage in a multistage $Q \Sigma \Delta M$ with complex-valued signals and coefficients.

the third and the latter stages, which operate in the denominator of the noise-shaping term. However, the leakage of the quantization noise of the earlier stages might be limiting achievable resolution in practice because of nonideal matching of the digital filters [33]. One way to combat this phenomenon is to use adaptive filters [34, 35].

\subsection{I/Q imbalance in multistage $Q \boldsymbol{\Sigma} \Delta \mathbf{M s}$}

In this section, a closed-form transfer function analysis is carried out for a general multistage $\mathrm{Q} \Sigma \Delta \mathrm{M}$ taking also the possible coefficient mismatches in complex I/Q signal processing into account. For mathematical tractability and notational convenience, second-order $\mathrm{Q} \Sigma \Delta \mathrm{M}$ stages are assumed as individual building blocks (individual stages) in Figure 4, and the purpose is to derive a complete closedform transfer function model for the overall multistage converter. Such analysis is missing from the existing stateof-the-art literature. For notational simplicity, the modulator coefficients are denoted in the following analysis as shown in the block diagram of Figure 5. With this structure, the ideal NTF for the $l$ th stage is given by

$$
\begin{aligned}
\operatorname{NTF}_{l}[z]= & \left(1-\left(M^{(l)}+N^{(l)}\right) z^{-1}+\left(M^{(l)} N^{(l)}\right) z^{-2}\right) \\
& /\left(1-\left(M^{(l)}+N^{(l)}+R^{(l)}\right) z^{-1}\right. \\
& \left.+\left(M^{(l)} N^{(l)}+N^{(l)} R^{(l)}-S^{(l)}\right) z^{-2}\right) .
\end{aligned}
$$

At the same time, the ideal STF for the $l$ th stage is defined as

$$
\begin{aligned}
\mathrm{STF}_{l}[z]= & \left(A^{(l)}+\left(B^{(l)}-N^{(l)} A^{(l)}-M^{(l)} A^{(l)}\right) z^{-1}\right. \\
& \left.+\left(C^{(l)}-N^{(l)} B^{(l)}+M^{(l)} N^{(l)} A^{(l)}\right) z^{-2}\right) \\
& /\left(1-\left(M^{(l)}+N^{(l)}+R^{(l)}\right) z^{-1}\right. \\
& \left.+\left(M^{(l)} N^{(l)}+N^{(l)} R^{(l)}-S^{(l)}\right) z^{-2}\right) .
\end{aligned}
$$

The transfer functions of (8) and (9) are valid when I and $\mathrm{Q}$ rails of the $\mathrm{Q} \Sigma \Delta \mathrm{M}$ are matched perfectly. With this perfect matching, (1) and (4) give the outputs for single-stage and multistage modulators, respectively.

\subsection{I/Q imbalance effects on individual $Q \Sigma \Delta M$ stage}

Quadrature signal processing is, in practice, implemented with parallel real signals and coefficients. In Figure 6, this is demonstrated in case of a single second-order $\mathrm{Q} \Sigma \Delta \mathrm{M}$ stage (parallel real I and Q signal rails) and taking possible mismatches in the coefficients into account. Deviation between coefficient values of the rails, which should ideally be the same, results in MFI. This interference can be presented mathematically with conjugate response of the signal and the noise components. Thus, image signal transfer function (ISTF) and image noise transfer function (INTF) are introduced, in addition to the traditional STF and NTF, to describe the output under I/Q imbalance. In the following, an analytic model is presented, first for individual stages of a multistage $\mathrm{Q} \Sigma \Delta \mathrm{M}$, and then for I/Q mismatched multistage $\mathrm{Q} \Sigma \Delta \mathrm{M}$, having arbitrary number of stages, as a whole. Such analysis has not been presented in the literature earlier.

The I/Q imbalance analysis for a single stage is based on the block diagram given in Figure 6. In this figure, real and imaginary parts of the coefficients of Figure 5 are marked with subscripts re and im, whereas nonideal implementation values of the signal rails are separated with subscripts 1 and 2 . The independent coefficients of the stages are denoted with superscript $l$. Thus, to obtain the complex outputs $V_{l}[z]=V_{\mathrm{I}, l}[z]+j V_{\mathrm{Q}, l}[z]$ of the stages $(l \in\{1, L\})$, the I branch outputs can be first shown to be

$$
\begin{aligned}
V_{\mathrm{I}, l}[z]= & \frac{\alpha_{\mathrm{I}}^{(l)}[z]}{\gamma_{\mathrm{I}}^{(l)}[z]} U_{\mathrm{I}, l}[z]-\frac{\beta_{\mathrm{I}}^{(l)}[z]}{\gamma_{\mathrm{I}}^{(l)}[z]} U_{\mathrm{Q}, l}[z] \\
& +\frac{\varepsilon_{\mathrm{I}}^{(l)}[z]}{\gamma_{\mathrm{I}}^{(l)}[z]} E_{\mathrm{I}, l}[z]+\frac{\eta_{\mathrm{I}}^{(l)}[z]}{\gamma_{\mathrm{I}}^{(l)}[z]} E_{\mathrm{Q}, l}[z] \\
& -\frac{\rho_{\mathrm{I}}^{(l)}[z]}{\gamma_{\mathrm{I}}^{(l)}[z]} V_{\mathrm{Q}, l}[z]
\end{aligned}
$$

where the auxiliary variables multiplying the signal components are defined by the coefficients (see Figure 6) in the 


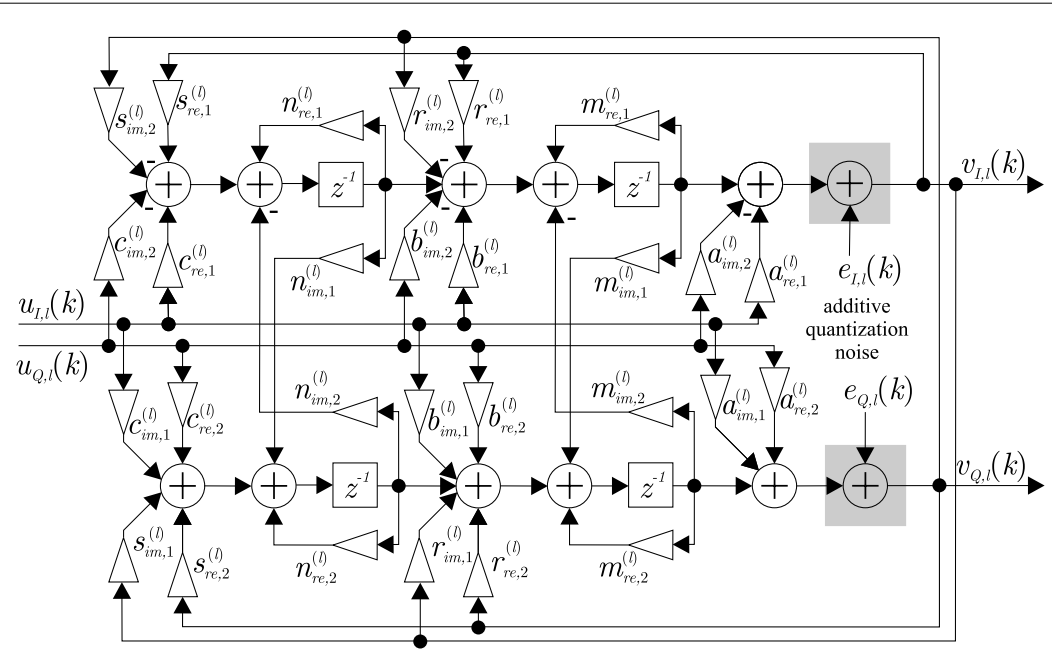

Figure 6 Implementation structure of the Ith second-order $Q \Sigma \Delta M$ stage in a multistage $Q \Sigma \Delta M$ with parallel real signals and coefficients taking possible mismatches into account.

following manner:

$$
\begin{aligned}
& \alpha_{\mathrm{I}}^{(l)}[z]= a_{\mathrm{re}, 1}^{(l)}+\left[b_{\mathrm{re}, 1}^{(l)}-m_{\mathrm{re}, 1}^{(l)} a_{\mathrm{re}, 1}^{(l)}-n_{\mathrm{re}, 1}^{(l)} a_{\mathrm{re}, 1}^{(l)}\right. \\
&\left.+n_{\mathrm{im}, 2}^{(l)} a_{\mathrm{im}, 1}^{(l)}+m_{\mathrm{im}, 2}^{(l)} a_{\mathrm{im}, 1}^{(l)}\right] z^{-1} \\
&+\left[c_{\mathrm{re}, 1}^{(l)}-n_{\mathrm{re}, 1}^{(l)} b_{\mathrm{re}, 1}^{(l)}+n_{\mathrm{re}, 1}^{(l)} m_{\mathrm{re}, 1}^{(l)} a_{\mathrm{re}, 1}^{(l)}\right. \\
&-n_{\mathrm{re}, 1}^{(l)} m_{\mathrm{im}, 2}^{(l)} a_{\mathrm{im}, 1}^{(l)}+n_{\mathrm{im}, 2}^{(l)} b_{\mathrm{im}, 1}^{(l)} \\
&\left.-n_{\mathrm{im}, 2}^{(l)} m_{\mathrm{im}, 1}^{(l)} a_{\mathrm{re}, 1}^{(l)}-n_{\mathrm{im}, 2}^{(l)} m_{\mathrm{re}, 2}^{(l)} a_{\mathrm{im}, 1}^{(l)}\right] z^{-2}, \\
& \beta_{\mathrm{I}}^{(l)}[z]= a_{\mathrm{im}, 2}^{(l)}+\left[b_{\mathrm{im}, 2}^{(l)}-n_{\mathrm{re}, 1}^{(l)} a_{\mathrm{im}, 2}^{(l)}-n_{\mathrm{im}, 2}^{(l)} a_{\mathrm{re}, 2}^{(l)}\right. \\
&\left.-m_{\mathrm{re}, 1}^{(l)} a_{\mathrm{im}, 2}^{(l)}-m_{\mathrm{im}, 2}^{(l)} a_{\mathrm{re}, 2}^{(l)}\right] z^{-1} \\
&+\left[c_{\mathrm{im}, 2}^{(l)}-n_{\mathrm{re}, 1}^{(l)} b_{\mathrm{im}, 2}^{(l)}+n_{\mathrm{re}, 1}^{(l)} m_{\mathrm{re}, 1}^{(l)} a_{\mathrm{im}, 2}^{(l)}\right. \\
&+n_{\mathrm{re}, 1}^{(l)} m_{\mathrm{im} 2,,}^{(l)} a_{\mathrm{re}, 2}^{(l)}-n_{\mathrm{im}, 2}^{(l)} b_{\mathrm{re}, 2}^{(l)} \\
&\left.-n_{\mathrm{im}, 2}^{(l)} m_{\mathrm{im}, 1}^{(l)} a_{\mathrm{im}, 2}^{(l)}+n_{\mathrm{im}, 2}^{(l)} m_{\mathrm{re}, 2}^{(l)} a_{\mathrm{re}, 2}^{(l)}\right] z^{-2}, \\
&-\left[s_{\mathrm{re}, 1}^{(l)}-n_{\mathrm{re}, 1}^{(l)} r_{\mathrm{re}, 1}^{(l)}+n_{\mathrm{im}, 2}^{(l)} r_{\mathrm{im}, 1}^{(l)}\right. \\
&\left.-n_{\mathrm{re}, 1}^{(l)} m_{\mathrm{re}, 1}^{(l)}+n_{\mathrm{im}, 2}^{(l)} m_{\mathrm{im}, 1}^{(l)}\right] z^{-2} . \\
& \varepsilon_{\mathrm{I}}^{(l)}[z]=1 {\left[n_{\mathrm{re}, 1}^{(l)}+m_{\mathrm{re}, 1}^{(l)}\right] z^{-1} } \\
&+\left[n_{\mathrm{re}, 1}^{(l)} m_{\mathrm{re}, 1}^{(l)}-n_{\mathrm{im}, 2}^{(l)} m_{\mathrm{im}, 1}^{(l)}\right] z^{-2}, \\
& \gamma_{\mathrm{I}}^{(l)}[z]=1-\left[n_{\mathrm{re}, 1}^{(l)} m_{\mathrm{im}, 2}^{(l)}-n_{\mathrm{im}, 2}^{(l)} m_{\mathrm{re}, 2}^{(l)}\right] z^{-2}, \\
& \rho_{\mathrm{I}}^{(l)}[z]=[\left.n_{\mathrm{im}, 2}^{(l)}+r_{\mathrm{im}, 2}^{(l)}+m_{\mathrm{im}, 2}^{(l)}\right] z^{-1} \\
&-\left[s_{\mathrm{im}, 2}^{(l)}-n_{\mathrm{re}, 1}^{(l)} r_{\mathrm{im}, 2}^{(l)}-n_{\mathrm{im}, 2}^{(l)} r_{\mathrm{re}, 2}^{(l)}\right. \\
&-\left[n_{\mathrm{re}, 1}^{(l)} m_{\mathrm{im}, 2}^{(l)}+n_{\mathrm{im}, 2}^{(l)} m_{\mathrm{re}, 2}^{(l)}\right] z^{-2}, \\
&\left.\eta_{\mathrm{im}, 2}\right] z^{-1} \\
&(l)
\end{aligned}
$$

This follows directly from a step-by-step signal analysis of the implementation structure in Figure 6. Similarly, the real-valued $Q$ branch outputs are given by

$$
\begin{aligned}
V_{\mathrm{Q}, l}[z]= & \frac{\beta_{\mathrm{Q}}^{(l)}[z]}{\gamma_{\mathrm{Q}}^{(l)}[z]} U_{\mathrm{I}, l}[z]+\frac{\alpha_{\mathrm{Q}}^{(l)}[z]}{\gamma_{\mathrm{Q}}^{(l)}[z]} U_{\mathrm{Q}, l}[z] \\
& +\frac{\varepsilon_{\mathrm{Q}}^{(l)}[z]}{\gamma_{\mathrm{Q}}^{(l)}[z]} E_{\mathrm{Q}, l}[z]-\frac{\eta_{\mathrm{Q}}^{(l)}[z]}{\gamma_{\mathrm{Q}}^{(l)}[z]} E_{\mathrm{I}, l}[z] \\
& +\frac{\rho_{\mathrm{Q}}^{(l)}[z]}{\gamma_{\mathrm{Q}}^{(l)}[z]} V_{\mathrm{I}, l}[z],
\end{aligned}
$$

where

$$
\begin{aligned}
\alpha_{\mathrm{Q}}^{(l)}[z]= & a_{\mathrm{re}, 2}^{(l)}+\left[b_{\mathrm{re}, 2}^{(l)}+n_{\mathrm{im}, 1}^{(l)} a_{\mathrm{im}, 2}^{(l)}-n_{\mathrm{re}, 2}^{(l)} a_{\mathrm{re}, 2}^{(l)}\right. \\
& \left.+m_{\mathrm{im}, 1}^{(l)} a_{\mathrm{im}, 2}^{(l)}-m_{\mathrm{re}, 2}^{(l)} a_{\mathrm{re}, 2}^{(l)}\right] z^{-1} \\
& +\left[c_{\mathrm{re}, 2}^{(l)}-n_{\mathrm{re}, 2}^{(l)} b_{\mathrm{re}, 2}^{(l)}-n_{\mathrm{im}, 1}^{(l)} m_{\mathrm{im}, 2}^{(l)} a_{\mathrm{re}, 2}^{(l)}\right. \\
& +n_{\mathrm{im}, 1}^{(l)} b_{\mathrm{im}, 2}^{(l)}-n_{\mathrm{im}, 1}^{(l)} m_{\mathrm{re}, 1}^{(l)} a_{\mathrm{im}, 2}^{(l)} \\
& \left.-n_{\mathrm{re}, 2}^{(l)} m_{\mathrm{im}, 1}^{(l)} a_{\mathrm{im}, 2}^{(l)}+n_{\mathrm{re}, 2}^{(l)} m_{\mathrm{re}, 2}^{(l)} a_{\mathrm{re}, 2}^{(l)}\right] z^{-2}, \\
\beta_{\mathrm{Q}}[z]= & a_{\mathrm{im}, 1}^{(l)}+\left[b_{\mathrm{im}, 1}^{(l)}-n_{\mathrm{im}, 1}^{(l)} a_{\mathrm{re}, 1}^{(l)}-n_{\mathrm{re}, 2}^{(l)} a_{\mathrm{im}, 1}^{(l)}\right. \\
& \left.-m_{\mathrm{im}, 1}^{(l)} a_{\mathrm{re}, 1}^{(l)}-m_{\mathrm{re}, 2}^{(l)} a_{\mathrm{im}, 1}^{(l)}\right] z^{-1} \\
& +\left[c_{\mathrm{im}, 1}^{(l)}-n_{\mathrm{re}, 2}^{(l)} b_{\mathrm{im}, 1}^{(l)}-n_{\mathrm{im}, 1}^{(l)} m_{\mathrm{im}, 2}^{(l)} a_{\mathrm{im}, 1}^{(l)}\right. \\
& -n_{\mathrm{im}, 1}^{(l)} b_{\mathrm{re}, 1}^{(l)}+n_{\mathrm{im}, 1}^{(l)} m_{\mathrm{re}, 1}^{(l)} a_{\mathrm{re}, 1}^{(l)} \\
& \left.+n_{\mathrm{re}, 2}^{(l)} m_{\mathrm{im}, 1}^{(l)} a_{\mathrm{re}, 1}^{(l)}+n_{\mathrm{re}, 2}^{(l)} m_{\mathrm{re}, 2}^{(l)} a_{\mathrm{im}, 1}^{(l)}\right] z^{-2}, \\
\varepsilon_{\mathrm{Q}}^{(l)}[z]= & -\left[n_{\mathrm{re}, 2}^{(l)}+m_{\mathrm{re}, 2}^{(l)}\right] z^{-1} \\
& +\left[n_{\mathrm{re}, 2}^{(l)} m_{\mathrm{re}, 2}^{(l)}-n_{\mathrm{im}, 1}^{(l)} m_{\mathrm{im}, 2}^{(l)}\right] z^{-2},
\end{aligned}
$$




$$
\begin{aligned}
\eta_{\mathrm{Q}}^{(l)}[z]= & {\left[n_{\mathrm{im}, 1}^{(l)}+m_{\mathrm{im}, 1}^{(l)}\right] z^{-1} } \\
& +\left[n_{\mathrm{im}, 1}^{(l)} m_{\mathrm{re}, 1}^{(l)}+n_{\mathrm{re}, 2}^{(l)} m_{\mathrm{im}, 1}^{(l)}\right] z^{-2}, \\
\rho_{\mathrm{Q}}^{(l)}[z]= & {\left[n_{\mathrm{re}, 2}^{(l)}+r_{\mathrm{re}, 2}^{(l)}+m_{\mathrm{re}, 2}^{(l)}\right] z^{-1} } \\
& +\left[s_{\mathrm{re}, 2}^{(l)}+n_{\mathrm{im}, 1}^{(l)} r_{\mathrm{im}, 2}^{(l)}-n_{\mathrm{re}, 2}^{(l)} r_{\mathrm{re}, 2}^{(l)}\right. \\
& \left.+n_{\mathrm{im}, 1}^{(l)} m_{\mathrm{im}, 2}^{(l)}-n_{\mathrm{re}, 2}^{(l)} m_{\mathrm{re}, 2}^{(l)}\right] z^{-2}, \\
\gamma_{\mathrm{Q}}^{(l)}[z]= & 1-\left[n_{\mathrm{im}, 1}^{(l)}+r_{\mathrm{im}, 1}^{(l)}+m_{\mathrm{im}, 1}^{(l)}\right] z^{-1} \\
& +\left[s_{\mathrm{im}, 1}^{(l)}-n_{\mathrm{im}, 1}^{(l)} r_{\mathrm{re}, 1}^{(l)}-n_{\mathrm{re}, 2}^{(l)} r_{\mathrm{im}, 1}^{(l)}\right. \\
& \left.-n_{\mathrm{im}, 1}^{(l)} m_{\mathrm{re}, 1}^{(l)}-n_{\mathrm{re}, 2}^{(l)} m_{\mathrm{im}, 1}^{(l)}\right] z^{-2} .
\end{aligned}
$$

In this way, the complex-valued output and the exact behavior of each transfer function can be solved analytically in different I/Q mismatch scenarios. As a result, the complex output of an individual stage with nonideal matching of the I and Q branches becomes

$$
\begin{aligned}
V_{l}[z]= & V_{\mathrm{I}, l}[z]+j V_{\mathrm{Q}, l}[z] \\
= & \operatorname{STF}_{l}[z] U_{l}[z]+\operatorname{ISTF}_{l}[z] U_{l}^{*}\left[z^{*}\right] \\
& +\operatorname{NTF}_{l}[z] E_{l}[z]+\operatorname{INTF}_{l}[z] E_{l}^{*}\left[z^{*}\right],
\end{aligned}
$$

where superscript asterisk (*) denotes complex conjugation, and the transfer functions are, based on (10) and (17) (omitting $[z]$ from the modulator coefficient variables of (11)-(16) and (18)-(23) for notational convenience), given by

$$
\begin{aligned}
\operatorname{STF}_{l}[z]= & \frac{\gamma_{Q}^{(l)} \alpha_{I}^{(l)}+\gamma_{I}^{(l)} \alpha_{Q}^{(l)}-\rho_{Q}^{(l)} \beta_{I}^{(l)}-\rho_{I}^{(l)} \beta_{Q}^{(l)}}{2\left(\gamma_{I}^{(l)} \gamma_{Q}^{(l)}+\rho_{I}^{(l)} \rho_{Q}^{(l)}\right)} \\
& +j \frac{\rho_{I}^{(l)} \alpha_{Q}^{(l)}+\rho_{Q}^{(l)} \alpha_{I}^{(l)}+\gamma_{Q}^{(l)} \beta_{I}^{(l)}+\gamma_{I}^{(l)} \beta_{Q}^{(l)}}{2\left(\gamma_{I}^{(l)} \gamma_{Q}^{(l)}+\rho_{I}^{(l)} \rho_{Q}^{(l)}\right)}, \\
\operatorname{ISTF}_{l}[z]= & \frac{\gamma_{Q}^{(l)} \alpha_{I}^{(l)}-\gamma_{I}^{(l)} \alpha_{Q}^{(l)}+\rho_{Q}^{(l)} \beta_{I}^{(l)}-\rho_{I}^{(l)} \beta_{Q}^{(l)}}{2\left(\gamma_{I}^{(l)} \gamma_{Q}^{(l)}+\rho_{I}^{(l)} \rho_{Q}^{(l)}\right)} \\
& +j \frac{\rho_{Q}^{(l)} \alpha_{I}^{(l)}-\rho_{I}^{(l)} \alpha_{Q}^{(l)}+\gamma_{I}^{(l)} \beta_{Q}^{(l)}-\gamma_{Q}^{(l)} \beta_{I}^{(l)}}{2\left(\gamma_{I}^{(l)} \gamma_{Q}^{(l)}+\rho_{I}^{(l)} \rho_{Q}^{(l)}\right)}, \\
\operatorname{NTF}_{l}[z]= & \frac{\gamma_{Q}^{(l)} \varepsilon_{I}^{(l)}+\gamma_{I}^{(l)} \varepsilon_{Q}^{(l)}+\rho_{I}^{(l)} \eta_{Q}^{(l)}+\rho_{Q}^{(l)} \eta_{I}^{(l)}}{2\left(\gamma_{I}^{(l)} \gamma_{Q}^{(l)}+\rho_{I}^{(l)} \rho_{Q}^{(l)}\right)} \\
& +j \frac{\rho_{I}^{(l)} \varepsilon_{Q}^{(l)}+\rho_{Q}^{(l)} \varepsilon_{I}^{(l)}-\gamma_{Q}^{(l)} \eta_{I}^{(l)}-\gamma_{I}^{(l)} \eta_{Q}^{(l)}}{2\left(\gamma_{I}^{(l)} \gamma_{Q}^{(l)}+\rho_{I}^{(l)} \rho_{Q}^{(l)}\right)}, \\
\operatorname{INTF}_{l}[z]= & \frac{\gamma_{Q}^{(l)} \varepsilon_{I}^{(l)}-\gamma_{I}^{(l)} \varepsilon_{Q}^{(l)}+\rho_{I}^{(l)} \eta_{Q}^{(l)}-\rho_{Q}^{(l)} \eta_{I}^{(l)}}{2\left(\gamma_{I}^{(l)} \gamma_{Q}^{(l)}+\rho_{I}^{(l)} \rho_{Q}^{(l)}\right)} \\
& +j \frac{\gamma_{Q}^{(l)} \eta_{I}^{(l)}-\gamma_{I}^{(l)} \eta_{Q}^{(l)}+\rho_{Q}^{(l)} \varepsilon_{I}^{(l)}-\rho_{I}^{(l)} \varepsilon_{Q}^{(l)}}{2\left(\gamma_{I}^{(l)} \gamma_{Q}^{(l)}+\rho_{I}^{(l)} \rho_{Q}^{(l)}\right)} .
\end{aligned}
$$

In Section 3.2, the above analysis for the individual stages $l \in\{1, L\}$ is combined to complete the closed-form overall model for the multistage $\mathrm{Q} \Sigma \Delta \mathrm{M}$.

Based on (24), the converter output consists of not only the (filtered) input signal and quantization noise but also their complex conjugates, which, in frequency domain, corresponds to spectral mirroring or imaging. Thus, based on (24), the so-called image rejection ratios (IRRs) of the $l$ th stage are

$$
\begin{aligned}
& \operatorname{IRR}_{\mathrm{STF}}^{(l)}\left[e^{j 2 \pi f T_{\mathrm{S}}}\right] \\
& \quad=10 \log _{10}\left(\left|\mathrm{STF}_{l}\left[e^{j 2 \pi f T_{\mathrm{S}}}\right]\right|^{2} /\left|\operatorname{STF}_{l}\left[e^{j 2 \pi f T_{\mathrm{S}}}\right]\right|^{2}\right)
\end{aligned}
$$

and

$$
\begin{aligned}
& \operatorname{IRR}_{\mathrm{NTF}}^{(l)}\left[e^{j 2 \pi f T_{\mathrm{S}}}\right] \\
& \quad=10 \log _{10}\left(\left|\mathrm{NTF}_{l}\left[e^{j 2 \pi f T_{S}}\right]\right|^{2} /\left|\operatorname{INTF}_{l}\left[e^{j 2 \pi f T_{S}}\right]\right|^{2}\right),
\end{aligned}
$$

where actual frequency-domain responses are attained with the substitution $z \leftarrow e^{j 2 \pi f T_{S}}$ to the earlier transfer functions, where $f$ is the frequency measured in Hertz and $T_{\mathrm{S}}$ is the sampling time. These IRR quantities describe the relation of the direct input signal and noise energy to the respective mismatch-induced MFI at the output signal. As an example, $\operatorname{IRR}_{\mathrm{STF}}^{(1)}\left(e^{j 2 \pi f_{0} T_{\mathrm{S}}}\right)=20 \mathrm{~dB}$ means that the power of the mismatch-induced (mirrored) conjugate input signal is $20 \mathrm{~dB}$ lower than the direct input signal at the frequency $f_{0}$. Similarly, $\operatorname{IRR}_{\mathrm{NTF}}^{(1)}\left(e^{j 2 \pi f_{0} T_{\mathrm{S}}}\right)=20 \mathrm{~dB}$ indicates that the nonconjugated quantization error level is $20 \mathrm{~dB}$ above the mirror image of the quantization error at the frequency $f_{0}$. Notice also that, in general, both IRRs are frequency-dependent functions.

\subsection{Combined I/Q imbalance effects of the stages in multistage $\mathrm{Q} \Sigma \Delta \mathrm{M}$}

For multistage $\mathrm{Q} \Sigma \Delta \mathrm{M}$, as illustrated in Figure 4, the final output signal is defined as a difference of digitally filtered output signals of the stages [33]. Furthermore, like shortly discussed already, the first-stage input $U_{1}[z]=U[z]$ while for $l>1, U_{l}[z]=E_{l-1}[z]$. The output of the first stage, given by (24) with $l=1$, is filtered with digital filter $H_{1}^{\mathrm{D}}[z]$ (usually matched to the STF of the second stage) and the output of the second stage, similarly given by (24) with $l=2$, is filtered with $H_{2}^{\mathrm{D}}[z]$ (usually matched to the NTF of the first stage), and so on for $l \in\{1, L\}$. Thus, the final output in case of I/Q mismatches in all the stages can now be expressed as

$$
V[z]=\sum_{l=1}^{L}(-1)^{l+1} H_{l}^{\mathrm{D}}[z] V_{l}[z] .
$$


Replacing $V_{l}[z]$ in (31) with (24) for $l \in\{1, L\}$ gives now an expression for the overall output as

$$
\begin{aligned}
V[z]= & \sum_{l=1}^{L}(-1)^{l+1} H_{l}^{\mathrm{D}}[z]\left(\mathrm{STF}_{l}[z] U_{l}[z]\right. \\
& +\operatorname{ISTF}_{l}[z] U_{l}^{*}\left[z^{*}\right]+\mathrm{NTF}_{l} E_{l}[z] \\
& \left.+\operatorname{INTF}_{l} E_{l}^{*}\left[z^{*}\right]\right),
\end{aligned}
$$

where the transfer functions are as defined in (25)-(28). Again, the digital filters are assumed matched to the analog transfer functions according to (6). As a concrete example, (32) can be evaluated for a three-stage $(L=3) \mathrm{Q} \Sigma \Delta \mathrm{M}$, giving

$$
\begin{aligned}
& V[z]=H_{1}^{\mathrm{D}}[z]\left(\operatorname{STF}_{1}[z] U[z]+\operatorname{ISTF}_{1}[z] U^{*}\left[z^{*}\right]\right. \\
& \left.+\mathrm{NTF}_{1} E_{1}[z]+\operatorname{INTF}_{1} E_{1}^{*}\left[z^{*}\right]\right) \\
& -H_{2}^{\mathrm{D}}[z]\left(\mathrm{STF}_{2}[z] E_{1}[z]+\operatorname{ISTF}_{2}[z] E_{1}^{*}\left[z^{*}\right]\right. \\
& \left.+\mathrm{NTF}_{2} E_{2}[z]+\operatorname{INTF}_{2} E_{2}^{*}\left[z^{*}\right]\right) \\
& +H_{3}^{\mathrm{D}}[z]\left(\mathrm{STF}_{3}[z] E_{2}[z]+\operatorname{ISTF}_{3}[z] E_{2}^{*}\left[z^{*}\right]\right. \\
& \left.+\mathrm{NTF}_{3} E_{3}[z]+\operatorname{INTF}_{3} E_{3}^{*}\left[z^{*}\right]\right) \\
& =\mathrm{STF}_{2}^{\mathrm{D}} \operatorname{STF}_{1}[z] U[z] \\
& +\operatorname{STF}_{2}^{\mathrm{D}} \operatorname{ISTF}_{1}[z] U^{*}\left[z^{*}\right] \\
& +\left(\mathrm{STF}_{2}^{\mathrm{D}}[z] \mathrm{NTF}_{1}[z]\right. \\
& \left.-\mathrm{NTF}_{1}^{\mathrm{D}}[z] \mathrm{STF}_{2}[z]\right) E_{1}[z] \\
& +\left(\mathrm{STF}_{2}^{\mathrm{D}}[z] \operatorname{INTF}_{1}[z]\right. \\
& \left.+\operatorname{NTF}_{1}^{\mathrm{D}}[z] \operatorname{ISTF}_{2}[z]\right) E_{1}^{*}\left[z^{*}\right] \\
& +\left(-\mathrm{NTF}_{1}^{\mathrm{D}}[z] \mathrm{NTF}_{2}[z]\right. \\
& +\left(\mathrm{NTF}_{1}^{\mathrm{D}}[z] \mathrm{NTF}_{2}^{\mathrm{D}}[z]\right. \\
& \left.\left./ \operatorname{STF}_{3}^{\mathrm{D}}[z]\right) \operatorname{STF}_{3}[z]\right) E_{2}[z] \\
& +\left(-\operatorname{NTF}_{1}^{\mathrm{D}}[z] \operatorname{INTF}_{2}[z]\right. \\
& +\left(\mathrm{NTF}_{1}^{\mathrm{D}}[z] \mathrm{NTF}_{2}^{\mathrm{D}}[z]\right. \\
& \left.\left./ \operatorname{STF}_{3}^{\mathrm{D}}[z]\right) \operatorname{ISTF}_{3}[z]\right) E_{2}^{*}\left[z^{*}\right] \\
& +\left(\mathrm{NTF}_{1}^{\mathrm{D}}[z] \mathrm{NTF}_{2}^{\mathrm{D}}[z] \mathrm{NTF}_{3}[z]\right. \\
& \left./ \mathrm{STF}_{3}^{\mathrm{D}}[z]\right) E_{3}[z] \\
& +\left(\mathrm{NTF}_{1}^{\mathrm{D}}[z] \mathrm{NTF}_{2}^{\mathrm{D}}[z] \mathrm{INTF}_{3}[z]\right. \\
& \left./ \operatorname{STF}_{3}^{\mathrm{D}}[z]\right) E_{3}^{*}\left[z^{*}\right] \\
& =\operatorname{STF}_{\mathrm{TOT}}[z] U[z]+\operatorname{ISTF}_{\mathrm{TOT}}[z] U^{*}\left[z^{*}\right] \\
& +\mathrm{NTF}_{\mathrm{TOT}, 1}[z] E_{1}[z]+\mathrm{INTF}_{\mathrm{TOT}, 1}[z] E_{1}^{*}\left[z^{*}\right] \\
& +\operatorname{NTF}_{\mathrm{TOT}, 2}[z] E_{2}[z]+\operatorname{INTF}_{\mathrm{TOT}, 2}[z] E_{2}^{*}\left[z^{*}\right] \\
& +\mathrm{NTF}_{\mathrm{TOT}, 3}[z] E_{3}[z]+\operatorname{INTF}_{\mathrm{TOT}, 3}[z] E_{3}^{*}\left[z^{*}\right]
\end{aligned}
$$

with digital filters $H_{1}^{\mathrm{D}}[z]=\operatorname{STF}_{2}^{\mathrm{D}}[z], H_{2}^{\mathrm{D}}[z]=\mathrm{NTF}_{1}^{\mathrm{D}}[z]$, and $H_{3}^{\mathrm{D}}[z]=\mathrm{NTF}_{1}^{\mathrm{D}}[z] \mathrm{NTF}_{2}^{\mathrm{D}}[z] / \mathrm{STF}_{3}^{\mathrm{D}}[z]$. It should be noted that $\mathrm{STF}_{\mathrm{TOT}}[z] U[z]$ and $\mathrm{NTF}_{\mathrm{TOT}, 3}[z] E_{3}[z]$ correspond structurally to the ideal output given in (7). However, the responses of $\mathrm{STF}_{\mathrm{TOT}}[z]$ and $\mathrm{NTF}_{\mathrm{TOT}, 3}[z]$ can be altered when compared to $\operatorname{STF}_{\mathrm{TOT}}^{\text {ideal }}[z]$ and $\operatorname{NTF}_{\mathrm{TOT}}^{\text {ideal }}[z]$ because of possible common-mode errors in the modulator coefficients [25]. Consequently, the six additional terms in (33) are considered as mismatch-induced interference, which includes the leakage of the first- and second-stage noises and the corresponding MFI (conjugate) components. It should also be noticed that the first-stage quantization error terms $\operatorname{STF}_{2}^{\mathrm{D}}[z] \mathrm{NTF}_{1}[z] E_{1}[z]$ and $\mathrm{NTF}_{1}^{\mathrm{D}}[z] \mathrm{STF}_{2}[z] E_{1}[z]$ do not reduce to zero because of noncommutativity of the complex transfer functions under I/Q imbalance [23]. On the other hand, secondstage quantization error vanishes if $\operatorname{NTF}_{1}^{\mathrm{D}}[z] \mathrm{NTF}_{2}[z]$ and $\left(\mathrm{NTF}_{1}^{\mathrm{D}}[z] \mathrm{NTF}_{2}^{\mathrm{D}}[z] / \mathrm{STF}_{3}^{\mathrm{D}}[z]\right) \mathrm{STF}_{3}[z]$ are equal. This means that $\operatorname{NTF}_{2}^{\mathrm{D}}[z]$ and $\operatorname{STF}_{3}^{\mathrm{D}}[z]$ should be equal to their analog counterparts, which can realized with, e.g., adaptive digital filters $[34,35]$. The matching can also be made more robust by designing the third stage to have unity signal response $\left(\mathrm{STF}_{3}[z]=1\right)$.

Now, based on (33), it is clear that filtered versions of the original and conjugate components of the input, the firststage, the second-stage, and the third-stage quantization errors all contribute to the final output. In order to inspect the overall IRR of the complete multistage structure, the transfer functions of the original signals (the input and the errors) and their conjugate counterparts should be compared. Based on (33), this gives the following formulas for the three-stage case considered herein:

$$
\begin{aligned}
& \operatorname{IRR}_{\mathrm{STF}_{\mathrm{TOT}}}\left[e^{j 2 \pi f T_{\mathrm{S}}}\right]=10 \log _{10}\left(\left|\mathrm{STF}_{\mathrm{TOT}}\left[e^{j 2 \pi f T_{\mathrm{S}}}\right]\right|^{2}\right. \\
& \left.\qquad /\left|\operatorname{ISTF}_{\mathrm{TOT}}\left[e^{j 2 \pi f T_{\mathrm{S}}}\right]\right|^{2}\right), \\
& \operatorname{IRR}_{\mathrm{NTF}_{\mathrm{TOT}, 1}}\left[e^{j 2 \pi f T_{\mathrm{S}}}\right] \\
& =10 \log _{10}\left(\left|\mathrm{NTF}_{\mathrm{TOT}, 1}\left[e^{j 2 \pi f T_{\mathrm{S}}}\right]\right|^{2}\right. \\
& \left.\quad /\left|\operatorname{INTF}_{\mathrm{TOT}, 1}\left[e^{j 2 \pi f T_{\mathrm{S}}}\right]\right|^{2}\right), \\
& \operatorname{IRR}_{\mathrm{NTF}_{\mathrm{TOT}, 2}\left[e^{j 2 \pi f T_{\mathrm{S}}}\right]}=10 \log _{10}\left(\left|\operatorname{NTF}_{\mathrm{TOT}, 2}\left[e^{j 2 \pi f T_{\mathrm{S}}}\right]\right|^{2}\right. \\
& \left.\quad /\left|\operatorname{INTF}_{\mathrm{TOT}, 2}\left[e^{j 2 \pi f T_{\mathrm{S}}}\right]\right|^{2}\right), \\
& \operatorname{IRR}_{\mathrm{NTF}}=10 \log _{10}\left(\mid \mathrm{NTF}_{\mathrm{TOT}, 3}\left[e^{j 2 \pi f T_{\mathrm{S}}}\right]\right. \\
& \left.\quad /\left|\operatorname{INTF}_{\mathrm{TOT}, 3}\left[e^{j 2 \pi f T_{\mathrm{S}}}\right]\right|^{2}\right) .
\end{aligned}
$$

In addition to the above IRRs, the performance of a nonideal $\mathrm{Q} \Sigma \Delta \mathrm{M}$ can be measured by the amount of total additional interference stemming from the implementation 
nonidealities. This can be expressed with interference rejection ratio $\Gamma$. In case of a three-stage $\mathrm{Q} \Sigma \Delta \mathrm{M}$, following from (33), the signal component (interference-free output) is defined as

$$
\sigma(k)=\operatorname{STF}_{\mathrm{TOT}}(k) * u(k)+\mathrm{NTF}_{\mathrm{TOT}, 3}(k) * e_{3}(k),
$$

where impulse responses of the STF and third-stage NTF are convolving the overall input and third-stage quantization error, respectively. At the same time, the total interference component (total additional interference caused by the nonidealities) is defined as

$$
\begin{aligned}
\tau(k)= & \operatorname{ISTF}_{\mathrm{TOT}}(k) * u^{*}(k)+\operatorname{NTF}_{\mathrm{TOT}, 1}(k) * e_{1}(k) \\
& +\operatorname{INTF}_{\mathrm{TOT}, 1}(k) * e_{1}^{*}(k) \\
& +\operatorname{NTF}_{\mathrm{TOT}, 2}(k) * e_{2}(k) \\
& +\operatorname{INTF}_{\mathrm{TOT}, 2}(k) * e_{2}^{*}(k) \\
& +\operatorname{INTF}_{\mathrm{TOT}, 3}(k) * e_{3}^{*}(k),
\end{aligned}
$$

where time-domain signal components are again convolved by respective transfer function impulseresponses. It should be noted that, in case of ideal three-stage $\mathrm{Q} \Sigma \Delta \mathrm{M}$, (39) reduces to zero. Now, interference rejection ratio at any given useful signal band is given by the integrals of spectral densities $G_{\sigma}\left(e^{j 2 \pi f T_{S}}\right)$ and $G_{\tau}\left(e^{j 2 \pi f T_{S}}\right)$ of the above random signals $\sigma(k)$ and $\tau(k)$, i.e.,

$$
\Gamma_{1}=\frac{\int_{f \in \Omega_{\mathrm{C}, 1}} G_{\sigma}\left(e^{j 2 \pi f T_{\mathrm{S}}}\right) d f}{\int_{f \in \Omega_{\mathrm{C}, 1}} G_{\tau}\left(e^{j 2 \pi f T_{\mathrm{S}}}\right) d f},
$$

where integration is done over the preferred signal band, defined as $\Omega_{\mathrm{C}, 1}=\left\{f_{\mathrm{C}, 1}-W_{1} / 2, \ldots, f_{\mathrm{C}, 1}+W_{1} / 2\right\}$ (where $W_{1}$ is the bandwidth of the signal). If there are two parallel signals (two-band scenario), the interference rejection ratio of the second signal is calculated in similar manner:

$$
\Gamma_{2}=\frac{\int_{f \in \Omega_{\mathrm{C}, 2}} G_{\sigma}\left(e^{j 2 \pi f T_{\mathrm{S}}}\right) d f}{\int_{f \in \Omega_{\mathrm{C}, 2}} G_{\tau}\left(e^{j 2 \pi f T_{\mathrm{S}}}\right) d f},
$$

where $\Omega_{\mathrm{C}, 2}=\left\{f_{\mathrm{C}, 2}-W_{2} / 2, \ldots, f_{\mathrm{C}, 2}+W_{2} / 2\right\}$.

An example of interference rejection ratio analysis in receiver-dimensioning context is given in Section 5. In addition, the roles of the separate signal components are further illustrated with numerical results in Section 6.

\section{$4 Q \Sigma \Delta M$ transfer function parametrization and design for $C R$ under I/Q imbalance}

In CR-type wideband receiver, signal dynamics can be tens of (even 50-60) dBs [5, 6]. With such signal composition, controlling linearity and image rejection of the receiver components is essential $[5,6,9]$. In this section, we concentrate on $\mathrm{Q} \Sigma \Delta \mathrm{M}$ transfer function design under I/Q imbalance, having minimization of input signal oriented MFI as the goal.

\subsection{Transfer function parametrization for reconfigurable CR receivers}

The NTF and STF of a Q $\Sigma \Delta \mathrm{M}$ can be designed by placing transfer function zeros and poles, parameterized and tuned (allowing reconfigurability) by the $\mathrm{Q} \Sigma \Delta \mathrm{M}$ coefficients, inside the unit circle [18]. In the following, the design process is described for a second-order $\mathrm{Q} \Sigma \Delta \mathrm{M}$ as a single-stage converter or an individual stage $l$ of a multistage converter. This is then extended to multistage converters in Section 4.2.

Based on the numerator of (8), the NTF zeros of the second-order $\mathrm{Q} \Sigma \Delta \mathrm{M}$ are defined by the loop-filter feedback coefficients, i.e.,

$$
\begin{aligned}
& \varphi_{\mathrm{NTF}, 1}^{(l)}=M^{(l)}=\lambda_{\mathrm{NTF}, 1}^{(l)} e^{j 2 \pi f_{\mathrm{NTF}, 1}^{(l)} T_{\mathrm{S}}}, \\
& \varphi_{\mathrm{NTF}, 2}^{(l)}=N^{(l)}=\lambda_{\mathrm{NTF}, 2}^{(l)} e^{j 2 \pi f_{\mathrm{NTF}, 2}^{(l)} T_{\mathrm{S}}},
\end{aligned}
$$

where $\lambda_{\mathrm{NTF}, 1}^{(l)}=\left|\varphi_{\mathrm{NTF}, 1}^{(l)}\right|$ and $\lambda_{\mathrm{NTF}, 2}^{(l)}=\left|\varphi_{\mathrm{NTF}, 2}^{(l)}\right|$, being usually set to unity for the zero-placement on the unit circle, and $f_{\mathrm{NTF}, 1}^{(l)}$ and $f_{\mathrm{NTF}, 2}^{(l)}$ are the frequencies of the two NTF notches. Thus, designing these complex gains tunable allows straightforward reconfigurability for NTF notch frequencies based on the spectrum-sensing information about the preferred information signals. Common choice is to place NTF zeros on the preferred signal band or in case of multi-band reception on those bands, generating the preferred noise-shaping effect. At the same time, the poles, which are common to the NTF and the STF, are solved based on the denominator of either (8) or (9), giving

$$
\begin{aligned}
\psi_{\text {common, } 1}^{(l)}= & \left(R^{(l)}+M^{(l)}+N^{(l)}+\left(R^{(l)^{2}}+M^{(l)^{2}}\right.\right. \\
& +N^{(l)^{2}}+2 R^{(l)} N^{(l)}-2 R^{(l)} M^{(l)} \\
& \left.\left.-2 M^{(l)} N^{(l)}+4 S^{(l)}\right)^{1 / 2}\right) / 2 \\
= & \lambda_{\text {pole }, 1}^{(l)} e^{j 2 \pi f_{\text {pole }, 1}^{(l)}}, \\
\psi_{\text {common }, 2}^{(l)}= & \left(R^{(l)}+M^{(l)}+N^{(l)}-\left(R^{(l)^{2}}+M^{(l)^{2}}\right.\right. \\
& +N^{(l)^{2}}+2 R^{(l)} N^{(l)} \\
& \left.\left.-2 R^{(l)} M^{(l)}-2 M^{(l)} N^{(l)}+4 S^{(l)}\right)^{1 / 2}\right) / 2 \\
= & \lambda_{\text {pole }, 2}^{(l)} e^{j 2 \pi f_{\text {pole }, 2}^{(l)}},
\end{aligned}
$$

where $\lambda_{\text {pole, } 1}^{(l)}=\left|\psi_{\text {common, } 1}^{(l)}\right|$ and $\lambda_{\text {pole, } 2}^{(l)}=\left|\psi_{\text {common, } 2}^{(l)}\right|$, which can be used to tune the magnitude of the poles and $f_{\mathrm{pole}, 1}^{(l)}$ 
and $f_{\text {pole }, 2}^{(l)}$, are the frequencies of the poles. The coefficients $M^{(l)}$ and $N^{(l)}$ are already fixed according to (42), leaving $R^{(l)}$ and $S^{(l)}$ free to tune the pole placement. The poles can, e.g., be placed on the frequency bands of the preferred signals to elevate the STF response and thus give gain for the preferred signals. However, the pole placement elevates also the STF response, and thus this kind of design is always a tradeoff between the noise-shaping and STF selectivity efficiencies.

On the other hand, the loop-filter coefficients $\left(M^{(l)}\right.$ and $N^{(l)}$ ) have also their effects on the STF zeros, which, however, can be further tuned with the input coefficients $\left(A^{(l)}\right.$, $B^{(l)}$, and $C^{(l)}$ ) of the modulator. This is illustrated in case of second-order $\mathrm{Q} \Sigma \Delta \mathrm{M}$, based on (9), by the expressions

$$
\begin{aligned}
\varphi_{\mathrm{STF}, 1}^{(l)}= & \left(1 / 2 A^{(l)}\right)\left(A^{(l)} M^{(l)}+A^{(l)} N^{(l)}-B^{(l)}\right) \\
& +\left(1 / 2 A^{(l)}\right)\left(B^{(l)^{2}}+A^{(l)^{2}} M^{(l)^{2}}+A^{(l)^{2}} N^{(l)^{2}}\right. \\
& +2 A^{(l)} B^{(l)} M^{(l)}-2 A^{(l)} B^{(l)} N^{(l)} \\
& \left.-2 A^{(l)} M^{(l)} N^{(l)}-4 A^{(l)} C^{(l)}\right)^{1 / 2} \\
= & \lambda_{\mathrm{STF}, 1}^{(l)} e^{j 2 \pi f_{\mathrm{STF}, 1}^{(l)}}, \\
\varphi_{\mathrm{STF}, 2}^{(l)}= & \left(1 / 2 A^{(l)}\right)\left(A^{(l)} M^{(l)}+A^{(l)} N^{(l)}-B^{(l)}\right) \\
& -\left(1 / 2 A^{(l)}\right)\left(B^{(l)^{2}}+A^{(l)^{2}} M^{(l)^{2}}+A^{(l)^{2}} N^{(l)^{2}}\right. \\
& +2 A^{(l)} B^{(l)} M^{(l)}-2 A^{(l)} B^{(l)} N^{(l)} \\
& \left.-2 A^{(l)} M^{(l)} N^{(l)}-4 A^{(l)} C^{(l)}\right)^{1 / 2} \\
= & \lambda_{\mathrm{STF}, 2}^{(l)} e^{j 2 \pi f_{\mathrm{STF}, 2}^{(l)} T_{\mathrm{S}}},
\end{aligned}
$$

where $\lambda_{\mathrm{STF}, 1}^{(l)}=\left|\varphi_{\mathrm{STF}, 1}^{(l)}\right|$ and $\lambda_{\mathrm{STF}, 2}^{(l)}=\left|\varphi_{\mathrm{STF}, 2}^{(l)}\right|$. Thus, (46)(47) clearly show that $A^{(l)}, B^{(l)}$, and $C^{(l)}$ allow independent placement of the STF zeros. In proportion to the NTF zero analysis above, $f_{\mathrm{STF}, 1}^{(l)}$ and $f_{\mathrm{STF}, 2}^{(l)}$ are the frequencies of the two STF notches. The proposed way to design the STF includes setting $f_{\mathrm{STF}, 1}^{(l)}$ and $f_{\mathrm{STF}, 2}^{(l)}$ to be the mirror frequencies of the preferred information signals (based on the spectrum-sensing information) to attenuate possible blockers on those critical frequency bands. More generally, these frequencies, and thus the STF zero locations, can be tuned to give preferred frequency-selective response for the STF. On the other hand, if frequency-flat STF design is preferred, then the zeros can be set to the origin by setting $\lambda_{\mathrm{STF}, 1}^{(l)}$ and $\lambda_{\mathrm{STF}, 2}^{(l)}$ to zero.

Usually, the first step in the Q $\Sigma \Delta \mathrm{M}$ NTF and STF design is to obtain the placements of the zeros and the poles as already discussed above. Thereafter, the modulator coefficient values realizing those zeros and poles should be found out. In the following, this procedure is explained for a second-order $\mathrm{Q} \Sigma \Delta \mathrm{M}$ as the $l$ th stage of a multistage $\mathrm{Q} \Sigma \Delta \mathrm{M}$. Practically, the goal is to find values for the input coefficients $\left(A^{(l)}, B^{(l)}\right.$, and $\left.C^{(l)}\right)$, the loop-filter coefficients
$\left(M^{(l)}\right.$ and $\left.N^{(l)}\right)$ and the feedback coefficients $\left(R^{(l)}\right.$ and $\left.S^{(l)}\right)$ that realize the STF zeros $\left(\varphi_{\mathrm{STF}, 1}^{(l)}\right.$ and $\left.\varphi_{\mathrm{STF}, 2}^{(l)}\right)$, the NTF ze$\operatorname{ros}\left(\varphi_{\mathrm{NTF}, 1}^{(l)}\right.$ and $\left.\varphi_{\mathrm{NTF}, 2}^{(l)}\right)$, and the common poles $\left(\psi_{\mathrm{common}, 1}^{(l)}\right.$ and $\psi_{\text {common, } 2}^{(l)}$ ) fixed above based on the transfer function characteristics.

The numerator of the NTF, the numerator of the STF, and the denominator of both transfer functions are used to solve the coefficient values. To begin with, the loop-filter feedback coefficients $M^{(l)}$ and $N^{(l)}$, the numerator of the NTF can be expressed with the modulator coefficients of the respective stage, as in (8), or with the help of the respective zeros $\varphi_{\mathrm{NTF}, 1}^{(l)}$ and $\varphi_{\mathrm{NTF}, 2}^{(l)}$. Setting these expressions equal, i.e.,

$$
\begin{aligned}
1 & -\left(M^{(l)}+N^{(l)}\right) z^{-1}+\left(M^{(l)} N^{(l)}\right) z^{-2} \\
& =1-\left(\varphi_{\mathrm{NTF}, 1}^{(l)}+\varphi_{\mathrm{NTF}, 2}^{(l)}\right) z^{-1}+\left(\varphi_{\mathrm{NTF}, 1}^{(l)} \varphi_{\mathrm{NTF}, 2}^{(l)}\right) z^{-2},
\end{aligned}
$$

allows for solving the coefficient values of the $l$ th stage based on the zeros by setting the terms with similar delays equal. Thus,

$$
\begin{aligned}
& M^{(l)}+N^{(l)}=\varphi_{\mathrm{NTF}, 1}^{(l)}+\varphi_{\mathrm{NTF}, 2}^{(l)}, \\
& M^{(l)} N^{(l)}=\varphi_{\mathrm{NTF}, 1}^{(l)} \varphi_{\mathrm{NTF}, 2}^{(l)},
\end{aligned}
$$

giving

$$
\begin{aligned}
& M^{(l)}=\varphi_{\mathrm{NTF}, 1}^{(l)}, \\
& N^{(l)}=\varphi_{\mathrm{NTF}, 2}^{(l)} .
\end{aligned}
$$

This result confirms that the NTF zeros are set by the complex-valued feedback gains of the loop integrators.

The input coefficients $A^{(l)}, B^{(l)}$, and $C^{(l)}$ of the $l$ th stage can be solved in similar manner, based on the STF numerator given in (9). Next, the numerator of (9) is set equal to the STF numerator presented with the respective zeros $\varphi_{\mathrm{STF}, 1}^{(l)}$ and $\varphi_{\mathrm{STF}, 2}^{(l)}$, i.e.,

$$
\begin{aligned}
A^{(l)}+ & \left(B^{(l)}-N^{(l)} A^{(l)}-M^{(l)} A^{(l)}\right) z^{-1} \\
+ & \left(C^{(l)}-N^{(l)} B^{(l)}+M^{(l)} N^{(l)} A^{(l)}\right) z^{-2} \\
& =1-\left(\varphi_{\mathrm{STF}, 1}^{(l)}+\varphi_{\mathrm{STF} .2}^{(l)}\right) z^{-1}+\left(\varphi_{\mathrm{STF}, 1}^{(l)} \varphi_{\mathrm{STF}, 2}^{(l)}\right) z^{-2} .
\end{aligned}
$$

Now, $A^{(l)}, B^{(l)}$, and $C^{(l)}$ can be solved setting the separate delay components equal. This gives

$$
\begin{aligned}
& A^{(l)}=1, \\
& B^{(l)}=N^{(l)} A^{(l)}+M^{(l)} A^{(l)}-\left(\varphi_{\mathrm{STF}, 1}^{(l)}+\varphi_{\mathrm{STF}, 2}^{(l)},\right. \\
& C^{(l)}=N^{(l)} B^{(l)}-M^{(l)} N^{(l)} A^{(l)}+\varphi_{\mathrm{STF}, 1}^{(l)} \varphi_{\mathrm{STF}, 2}^{(l)},
\end{aligned}
$$


pronouncing that these coefficient can be used to tune the STF response. However, the NTF zeros should also be taken indirectly into account because they define the values of $M^{(l)}$ and $N^{(l)}$, as found out in (51)-(52).

At this point, only the feedback coefficients $R^{(l)}$ and $S^{(l)}$ of the $l$ th stage remain unknown. Those can be solved using the common denominator of the NTF and the STF in (8) and (9). Again, the denominator of (8) and (9) is set equal to the denominator presented with the common poles of the transfer functions $\psi_{\text {common,1 }}^{(l)}$ and $\psi_{\text {common, } 2}^{(l)}$. In other words,

$$
\begin{aligned}
1- & \left(M^{(l)}+N^{(l)}+R^{(l)}\right) z^{-1} \\
+( & \left.M^{(l)} N^{(l)}+N^{(l)} R^{(l)}-S^{(l)}\right) z^{-2} \\
= & 1-\left(\psi_{\text {common }, 1}^{(l)}+\psi_{\text {common }, 2}^{(l)}\right) z^{-1} \\
& +\left(\psi_{\text {common, } 1}^{(l)} \psi_{\text {common }, 2}^{(l)} z^{-2} .\right.
\end{aligned}
$$

Again, setting the separate delay components equal gives solutions for the feedback coefficients:

$$
\begin{aligned}
& R^{(l)}=-M^{(l)}-N^{(l)}+\psi_{\text {common }, 1}^{(l)}+\psi_{\text {common, } 2}^{(l)}, \\
& S^{(l)}=M^{(l)} N^{(l)}+N^{(l)} R^{(l)}-\psi_{\text {common, } 1}^{(l)} \psi_{\text {common, } 2}^{(l)} .
\end{aligned}
$$

Thus, the feedback gains are affected by the NTF zeros (again via $M^{(l)}$ and $N^{(l)}$ ) but finally defined by the poles of both the transfer functions.

Based on this parametrization, tuning the modulator response in frequency agile way is straightforward. The spectrum-sensing information is used to extract the information about the frequency bands preferred to be received, and NTF zeros are placed on these frequencies $\left(f_{\mathrm{NTF}, 1}^{(l)}\right.$ and $f_{\mathrm{NTF}, 2}^{(l)}$ in second-order case) with unity magnitude $\left(\lambda_{\mathrm{NTF}, 1}^{(l)}=1\right.$ and $\lambda_{\mathrm{NTF}, 2}^{(l)}=1$ in second-order case). In addition, the most harmful blockers can be identified based on the spectrum sensing. Thus, the STF zeros can be set on the unit circle $\left(\lambda_{\mathrm{STF}, 1}^{(l)}=1\right.$ and $\lambda_{\mathrm{STF}, 2}^{(l)}=1$ in secondorder case) on the frequencies of those blocker signals $\left(f_{\mathrm{STF}, 1}^{(l)}\right.$ and $f_{\mathrm{STF}, 2}^{(l)}$ in second-order case). The poles can be used to tune both the transfer functions, being common though. Usually, the frequencies that are attenuated in the NTF design are supposed not to be attenuated in the STF and vice versa. This sets an optimization problem for the pole placement. Pole placement in the origin is of course a neutral choice. The authors have chosen poles on the preferred signal center frequencies, i.e., $f_{\mathrm{pole}, 1}^{(l)}=f_{\mathrm{NTF}, 1}^{(l)}$ and $f_{\mathrm{pole}, 2}^{(l)}=f_{\mathrm{NTF}, 2}^{(l)}$, to highlight STF selectivity with gain on the preferred signal bands. The magnitudes of the poles are chosen to be $\lambda_{\text {pole }, 1}^{(l)}=0.5$ and $\lambda_{\text {pole }, 2}^{(l)}=0.5$, thus pulling the poles half way off the unit circle to maintain efficient quantization noise shaping. A summary table of the overall de- sign flow will be presented, after discussing the design aspects under I/Q imbalance, at the end of the following subchapter.

\subsection{Multistage $Q \Sigma \Delta M$ transfer function design under I/Q imbalance}

In $\mathrm{Q} \Sigma \Delta \mathrm{Ms}$, the modulator feedback branch mismatches have been considered most crucial $[23,26,28]$. Exactly this problem can be fought against with mirror-frequencyrejecting STF design in a single-stage $\mathrm{Q} \Sigma \Delta \mathrm{M}$ [19] or in the first stage of multistage $\mathrm{Q} \Sigma \Delta \mathrm{M}$ [21]. The signal fed to the feedback branch of the modulator is the same as in the output, so the STF and NTF effects are seen therein in full extent. Considering this together with potential blocking signal energy on the mirror band, mirror-frequency-rejecting STF design is a recommended choice for feedback branchmismatched Q $\Sigma \Delta$ Ms based on the analysis in $[19,21]$.

The main difference in this design compared to the one proposed in [18] is deeper notching of the mirrorband(s) to attenuate possible input blocker(s) as effectively as possible. This is attained by setting the STF zeros on the unit-circle at the mirror-frequencies of the preferred information signals, meaning in second-order case that $\varphi_{\mathrm{STF}, 1}^{(1)}=\lambda_{\mathrm{STF}, 1}^{(1)} e^{j 2 \pi f_{\mathrm{STF}, 1}^{(1)} T_{\mathrm{S}}}=e^{-j 2 \pi f_{\mathrm{NTF}, 1}^{(1)} T_{\mathrm{S}}}$ and $\varphi_{\mathrm{STF}, 2}^{(1)}=$ $\lambda_{\mathrm{STF}, 2}^{(1)} e^{j 2 \pi f_{\mathrm{STF}, 2}^{(1)} T_{\mathrm{S}}}=e^{-j 2 \pi f_{\mathrm{NTF}, 2}^{(1)} T_{\mathrm{S}}}$, while the NTF zeros are located on the unit-circle $\left(\lambda_{\mathrm{NTF}, 1}^{(1)}=1\right.$ and $\left.\lambda_{\mathrm{NTF}, 1}^{(1)}=1\right)$ at $\varphi_{\mathrm{NTF}, 1}^{(1)}=e^{j 2 \pi f_{\mathrm{NTF}, 1}^{(1)} T_{\mathrm{S}}}$ and $\varphi_{\mathrm{NTF}, 2}^{(1)}=e^{j 2 \pi f_{\mathrm{NTF}, 2}^{(1)} T_{\mathrm{S}}}$. The poles are placed on the preferred signal center frequencies, as described above, to elevate the STF response, i.e., $\psi_{\text {common, } 1}^{(1)}=$ $0.5 e^{j 2 \pi f_{\mathrm{NTF}, 1}^{(1)} T_{\mathrm{S}}}$ and $\psi_{\text {common }, 2}^{(1)}=0.5 e^{j 2 \pi f_{\mathrm{NTF}, 2}^{(1)} T_{\mathrm{S}}}$ (with $\lambda_{\text {pole }, 1}^{(1)}=$ 0.5 and $\lambda_{\text {pole }, 2}^{(1)}=0.5$ ).

In multistage $\mathrm{Q} \Sigma \Delta \mathrm{Ms}$, the latter stages process only the quantization error of the preceding stage, and thus the STFs of these stages do not contribute to the overall inputoutput STF. This can be seen also in (7), where the overall STF is a product of the first-stage STF and the following digital $H_{1}^{\mathrm{D}}[z]$ filter matched to the STF of the second stage. From the signal-component point of view, the role of the first stage is emphasized because of the possible blockers in the input. The input of the latter stage(s) is the error of the previous stage and thus likely having less power variations along frequency axis. Albeit the overall STF is a product of the first two stage STFs, only the first-stage STF can offer robustness against input signal originating MFI stemming from the mismatches in the feedback branch of the first stage. Thus, design of the first-stage STF should be considered carefully in the presence of $\mathrm{I} / \mathrm{Q}$ mismatches. With second-order first stage, it is possible to place two zeros in the related (first-stage) STF and thus the design is constrained to rejection of two frequency bands from the MFI mitigation point of view. At the same time, the overall noise-shaping order is of the combined order of 
all the $L$ stages. Thus, the order of the first stage is limiting the capabilities to implement the mirror-frequencyrejecting STF design, e.g., in multi-band reception. The benefits of mirror-frequency-rejecting STF design will be demonstrated graphically and numerically in Section 6 using the earlier closed-form response analysis results and computer simulations.

Considering the NTF design of the stages under I/Q imbalance (a three-stage $\mathrm{Q} \Sigma \Delta \mathrm{M}$ used as an example), the role of the digital second-stage filter $H_{2}^{\mathrm{D}}[z]=\mathrm{NTF}_{1}^{\mathrm{D}}[z]$ is emphasized. In ideal case, the overall noise present at the output should be the noise of the last stage shaped by the product of all the stage NTFs. Thus, notching of each of the preferred signal frequency bands could be done in any of the stages having similar overall effect. However, under I/Q imbalance, quantization errors of the stages have also image response components, e.g., $\mathrm{NTF}_{1}^{\mathrm{D}}[z] \operatorname{ISTF}_{2}[z]$ for $E_{1}^{*}[z]$ and $-\mathrm{NTF}_{1}^{\mathrm{D}}[z] \operatorname{INTF}_{2}[z]$ for $E_{2}^{*}[z]$ (see (33)). Naturally, these terms are preferred to be minimized on all the interesting frequency bands. Thus, it is proposed to place the NTF zeros of the first stage at the center frequencies of the preferred information signals, i.e., $\varphi_{\mathrm{NTF}, 1}^{(1)}=e^{j 2 \pi f_{\mathrm{C}, 1} T_{\mathrm{S}}}$ and $\varphi_{\mathrm{NTF}, 2}^{(1)}=e^{j 2 \pi f_{\mathrm{C}, 2} T_{\mathrm{S}}}$, where $f_{\mathrm{C}, 1}$ and $f_{\mathrm{C}, 2}$ are the center frequencies of the two signals to be received. With the latter stage(s), the noise notches can widened by placing the respective NTF zeros around the ones of the first-stage NTF. This means that, e.g., in three-stage scenarios, the second-stage zeros are $\varphi_{\mathrm{NTF}, 1}^{(2)}=e^{j 2 \pi\left(f_{\mathrm{C}, 1} \pm f_{\text {offset }, 1}\right) T_{\mathrm{S}}}$ and $\varphi_{\mathrm{NTF}, 2}^{(2)}=e^{j 2 \pi\left(f_{\mathrm{C}, 2} \pm f_{\mathrm{offset}, 2}\right) T_{\mathrm{S}}}$ and for the third stage $\varphi_{\mathrm{NTF}, 1}^{(3)}=e^{j 2 \pi\left(f_{\mathrm{C}, 1} \mp f_{\text {offset }, 1}\right) T_{\mathrm{S}}}$ and $\varphi_{\mathrm{NTF}, 2}^{(3)}=$ $e^{j 2 \pi\left(f_{\mathrm{C}, 2} \mp f_{\text {offset }, 2}\right) T_{\mathrm{S}}}$, where $f_{\text {offset }, 1}=\sqrt{3 / 20} W_{1}$ and $f_{\text {offset }, 2}=$ $\sqrt{3 / 20} W_{2}$ ( $W_{1}$ and $W_{2}$ being the respective signal bandwidths) for optimal zero placements [36]. The signs in the exponent terms are opposite for the second- and the thirdstage zeros. The ideal model would allow also for such NTF design that the noise shaping of the interesting frequency bands would be done separately in different stages, meaning, e.g., that the first-stage NTF would notch the frequencies of certain signal and the second-stage NTF the frequencies of the other one. However, this kind of approach would allow the underlying I/Q imbalance-induced image components to leak more heavily on the latter signal band. The above-mentioned NTF design is proposed to avoid this scenario. This overall design flow, starting with spectrum-sensing information in terms of preferred signal center frequencies and blocker center frequencies, is illustrated as a whole in Table 1.

\section{Receiver system level considerations}

In this section, system level parameters are considered to define realistic target values for the needed interference rejection ratio introduced in Section 3. The proposed $\mathrm{Q} \Sigma \Delta \mathrm{M}$ performance is illustrated in a realistic multi-band

Table 1 Overall design flow of a three-stage two-band $Q \Sigma \Delta M$.

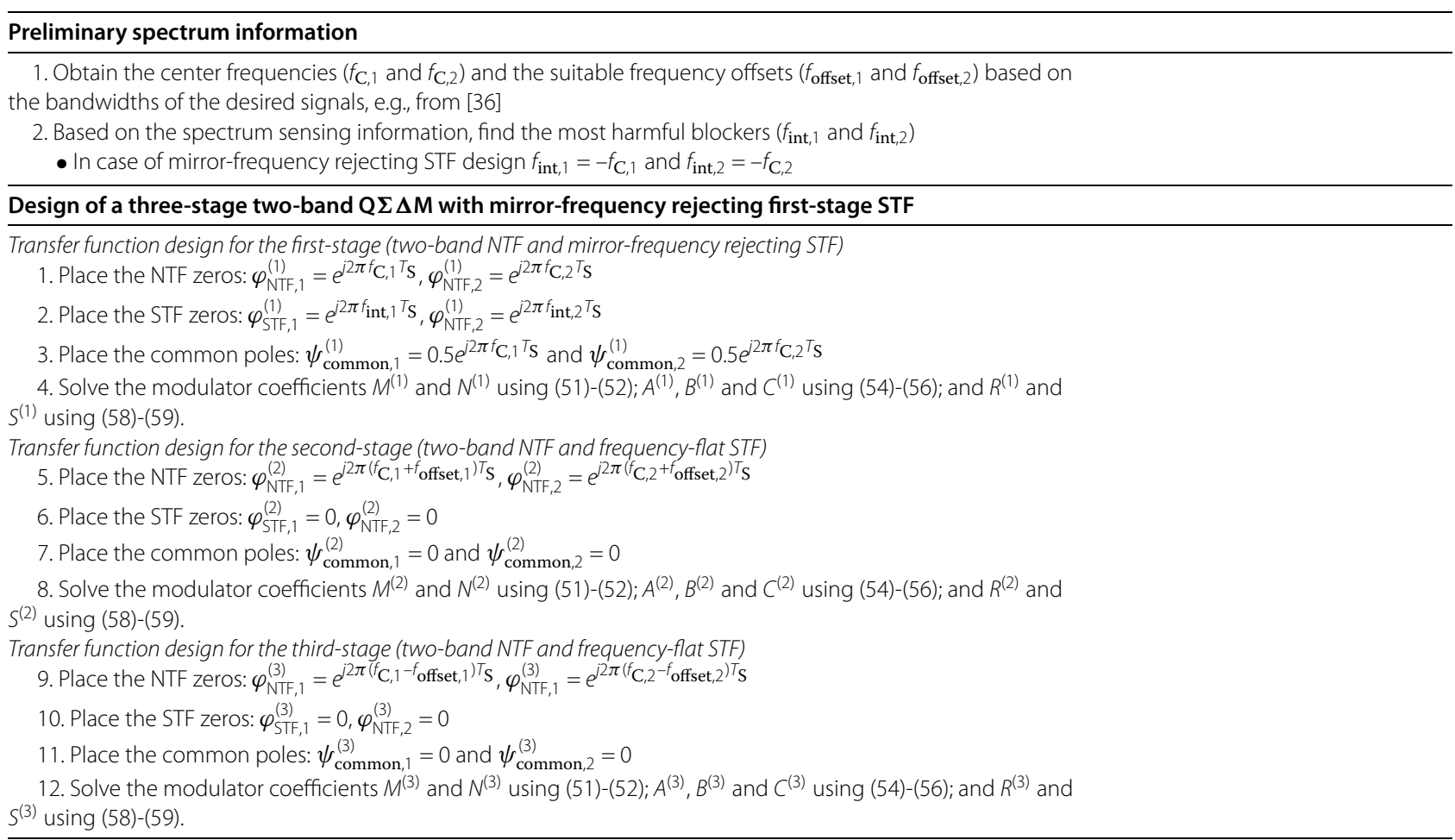


reception scheme, assuming the sampling frequency $f_{\mathrm{S}}=$ $128 \mathrm{MHz}$.

The detection of a 16-QAM waveform on intermediate frequency $f_{\mathrm{C}, 16-\mathrm{QAM}}=36.74 \mathrm{MHz}$ with bandwidth of $W_{16-\mathrm{QAM}}=10 \mathrm{MHz}$ is considered as a practical example. The received preferred signal power is assumed to be $-84 \mathrm{dBm}$ (sensitivity level), remaining $20 \mathrm{~dB}$ above the thermal noise floor at $-104 \mathrm{dBm}$. Taking typical receiver overall noise figure of $7 \mathrm{~dB}$ into account, this gives signalto-noise ratio (SNR) of $13 \mathrm{~dB}$ at the input of the ADC $\left(\mathrm{SNR}_{\mathrm{PRE}}=13 \mathrm{~dB}\right)$. Thus, with digital signal-to-interference and noise ratio (SINR) target of say $10 \mathrm{~dB}\left(\mathrm{SINR}_{\text {target }}=\right.$ $10 \mathrm{~dB}$ ) for detection, implementation margin of $3 \mathrm{~dB}$ is allowed.

Different combinations of $\mathrm{Q} \Sigma \Delta \mathrm{M}$ parameters are considered to highlight the flexibility of the structure, namely first-, third-, and sixth-order, noise shaping $\left(P_{16-\mathrm{QAM}}=\right.$ $\{1,3,6\})$ is applied for the preferred signal band in 1 - and 3bit quantization schemes $\left(b_{\mathrm{Q}}=\{1,3\}\right)$. The noise-shaping order $P_{16-\mathrm{QAM}}$ describes the combined noise-shaping effects of all the $\mathrm{Q} \Sigma \Delta \mathrm{M}$ stages on that frequency band according to the discussion in Sections 2 and 4, assuming an ideal $\mathrm{Q} \Sigma \Delta \mathrm{M}$. The related zero-optimization gains for each noise-shaping order $\left(\mathrm{ZOG}_{\mathrm{dB}}=\{0,8,23\}\right)$ are obtained from [36] and represent the SNR gain of the optimal zero placements compared to the case where all the zeros are on the center frequency of the preferred signal.

The Crest factors in the range of 4 to $6 \mathrm{~dB}$ were found in simulations with realistic power levels for a number of out-of-band signals in addition to the preferred one, depending on the exact power distribution (the simulation setup will be further discussed in Section 6). Thus, Crest factor of $\mathrm{CF}_{\mathrm{dB}}=5 \mathrm{~dB}$ is assumed in the following analysis for the sake of simplicity. The full-band signal power to the preferred signal power ratio is assumed to range from $0 \mathrm{~dB}$ (only the preferred signal) up to $+140 \mathrm{~dB}$. Such a high maximum value is chosen to illustrate also the performance of the sixth-order efficient noise shaping. The properties defined above are summarized in Table 2.

Based on the given parameters, signal-to-quantization noise ratio (SQNR) of the $\mathrm{Q} \Sigma \Delta \mathrm{M}$, yet without implementation nonidealities, can be solved in different scenarios by varying the amount of quantization bits and noise-shaping order. The SQNR equations derived for real lowpass modulators [13] of corresponding order can be adopted to use also in quadrature bandpass case because the noise-shaping efficiency is maintained with only asymmetric shift of the NTF notch center frequency. Thus, the inband SQNR for a single-frequency channel (assuming an ideal $\mathrm{Q} \Sigma \Delta \mathrm{M}$ ), taking also receiver out-of-band signal content into account, is defined as

$$
\begin{aligned}
\mathrm{SQNR}= & 6.02 b_{\mathrm{Q}}+4.76-\mathrm{CF}_{\mathrm{dB}} \\
& -10 \log _{10}\left(\frac{\pi^{2 P_{16-\mathrm{QAM}}}}{2 P_{16-\mathrm{QAM}}+1}\right) \\
& +\left(20 P_{16-\mathrm{QAM}}+10\right) \log _{10}\left(\frac{f_{\mathrm{S}}}{W_{16-\mathrm{QAM}}}\right) \\
& +\mathrm{ZOG}_{\mathrm{dB}}-10 \log _{10}\left(\frac{S_{\text {full-band }}}{S_{16-\mathrm{QAM}}}\right),
\end{aligned}
$$

where, in addition to the values given in Table $2, S_{\text {full-band }}$ is the power of the whole ADC input signal, and $S_{16-\text { QAM }}$ is the power of the preferred 16-QAM waveform. Increasing full-band signal power compared to the preferred signal power decreases the SQNR because with large values of this ratio, the out-of-band signal content dominates the dynamics of the overall signal. In this kind of scenario, the weak preferred signal is effectively scaled down at the ADC input. Now, the total SNR after the A/D conversion $\left(\mathrm{SNR}_{\mathrm{TOT}}\right)$ is the ratio of signal power $S_{16-\mathrm{QAM}}$ to the combined inband thermal noise power $N_{\text {PRE }}$ and inband quantization noise power $N_{\mathrm{Q}}\left(N_{\mathrm{TOT}}=N_{\mathrm{PRE}}+N_{\mathrm{Q}}\right)$. Furthermore, this ratio can be defined with $\mathrm{SNR}_{\mathrm{PRE}}$ and SQNR, giving

$$
\begin{aligned}
\mathrm{SNR}_{\mathrm{TOT}} & =10 \log _{10}\left(\frac{S_{16-\mathrm{QAM}}}{N_{\mathrm{TOT}}}\right) \\
& =10 \log _{10}\left(\frac{S_{16-\mathrm{QAM}}}{N_{\mathrm{PRE}}+N_{\mathrm{Q}}}\right) \\
& =10 \log _{10}\left(\frac{1}{10^{-\mathrm{SNR}_{\mathrm{PRE}} / 10}+10^{-\mathrm{SQNR} / 10}}\right) .
\end{aligned}
$$

\begin{tabular}{|c|c|c|c|}
\hline System properties & Value & A/D interface properties & Value \\
\hline Desired signal waveform & 16-QAM & Sampling frequency $f_{\mathrm{S}}$ & $128 \mathrm{MHz}$ \\
\hline Intermediate frequency $f_{\mathrm{C}, 16-\mathrm{QAM}}$ & $36.74 \mathrm{MHz}$ & Quantization bits $b_{\mathrm{Q}}$ & $\{1,3\}$ \\
\hline Desired signal bandwidth $W_{16-\mathrm{QAM}}$ & $10 \mathrm{MHz}$ & Noise-shaping order $P_{16-Q A M}$ & $\{1,3,6\}$ \\
\hline Received preferred signal power & $-84 \mathrm{dBm}$ & Zero-optimization gain $\mathrm{ZOG}_{\mathrm{dB}}$ & $\{0,8,23\} \mathrm{dB}$ \\
\hline Thermal noise $k T W_{16-Q A M}$ & $-104 \mathrm{dBm}$ & SNR $_{\text {PRE }}$ at the ADC input & $13 \mathrm{~dB}$ \\
\hline Receiver overall noise figure & $7 \mathrm{~dB}$ & Full-band Crest-factor $\mathrm{CF}_{\mathrm{dB}}$ & $5 \mathrm{~dB}(4 \ldots 6 \mathrm{~dB})$ \\
\hline Implementation margin & $3 \mathrm{~dB}$ & Full-band signal power relative to & 0 to $140 \mathrm{~dB}$ \\
\hline SINR $_{\text {target }}$ for detection & $10 \mathrm{~dB}$ & the desired signal power & \\
\hline
\end{tabular}

In addition, $\mathrm{SINR}_{\text {target }}$ set for the detection defines also the maximum level of additional inband interference com-

Table 2 A summary of receiver system level and A/D interface properties used in the interference rejection example. 
ponents other than thermal and quantization noises, such as MFI and noise leakage, generated by the $\Sigma \Delta$ modulator nonidealities, discussed in Section 3. In that section, interference rejection ratio $\Gamma$ was defined to measure the amount of this interference relative to the ideal modulator output inband power. Now, the maximum tolerable amount of additional inband interference $I_{\mathrm{MAX}}$, compared to the preferred signal, the inband thermal noise and the inband quantization noise powers $\left(S_{16-\mathrm{QAM}}+N_{\mathrm{TOT}}\right)$, defines the needed interference rejection ratio demanded to

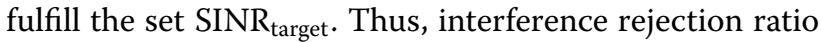
is given by

$$
\begin{aligned}
\Gamma_{\text {demand }} & =10 \log _{10}\left(\frac{S_{16-\mathrm{QAM}}+N_{\mathrm{TOT}}}{I_{\mathrm{MAX}}}\right) \\
& =10 \log _{10}\left(\frac{1+10^{-\mathrm{SNR}_{\mathrm{TOT}} / 10}}{10^{-\mathrm{SINR}_{\text {target }} / 10}-10^{-\mathrm{SNR}_{\mathrm{TOT}} / 10}}\right), \\
\mathrm{SNR}_{\mathrm{TOT}} & >\mathrm{SINR}_{\text {target }}
\end{aligned}
$$

If $\mathrm{SNR}_{\mathrm{TOT}}$ is below $\mathrm{SINR}_{\text {target }}$, achieving the set SINR is obviously not possible and a logarithm of a negative number results in a complex-valued $\Gamma_{\text {demand }}$ (hence the condition $\mathrm{SNR}_{\mathrm{TOT}}>\mathrm{SINR}_{\text {target }}$ ).

This interference rejection demand is plotted in Figure 7 as a function of the full-band signal power compared to the preferred inband signal power. The increasing power ratio on the $x$-axis limits the performance of the ADC because of the decreasing SQNR according to (60). Subsequently, from (62) it is clear that, if $\mathrm{SNR}_{\mathrm{TOT}}$ approaches SINR $_{\text {target, }}$ then the denominator goes to zero and thus $\Gamma_{\text {demand }}$ goes to infinity, indicating that no additional interference is allowed. The flooring at approximately $13 \mathrm{~dB}$ happens because, this is, together with the thermal noise SNR of $13 \mathrm{~dB}$, the minimum level of interference rejection ratio with any SQNR to achieve the SINR target of $10 \mathrm{~dB}$. The six $\mathrm{Q} \Sigma \Delta \mathrm{M}$ scenarios with 1- or 3-bit quantization and differing noise-shaping orders on the preferred signal band defined above are illustrated in Figure 7 as examples. The most straightforward case for multi-band reception of parallel signals with the bandwidths in megahertz-range is third-order noise shaping with 1- or 3-bit quantization, allowing two signal bands to be converted efficiently. These results are plotted with dashed lines and show tolerance of the full-band power to signal power ratios up to the range of 50 to $70 \mathrm{~dB}$, depending on the quantization scheme. The first- and sixth-order noise shapings are applicable for the conversion of narrow- and wideband signals, respectively. However, the results given in Figure 7 are applicable only with given exemplary set of parameters (see Table 2), such as $10-\mathrm{MHz}$ bandwidth. The derived interference rejection ratio demands are compared to the simulated achievable figures of the proposed $\mathrm{Q} \Sigma \Delta \mathrm{M}$ design in Section 6.

\section{Results and illustrations}

In this section, the models derived in Section 3 and the design principles in Section 4 are used to analytically calculate and illustrate the transfer functions for a threestage $\mathrm{Q} \Sigma \Delta \mathrm{M}$ under I/Q imbalance (Section 6.1). Finally, the $\mathrm{Q} \Sigma \Delta \mathrm{M}$ behavior under I/Q imbalance is simulated to illustrate the interference rejection performance of the modulator (Section 6.2) for which the target values were derived in Section 5.

In general, multi-band IF reception [19] of two parallel information signals around center frequencies of

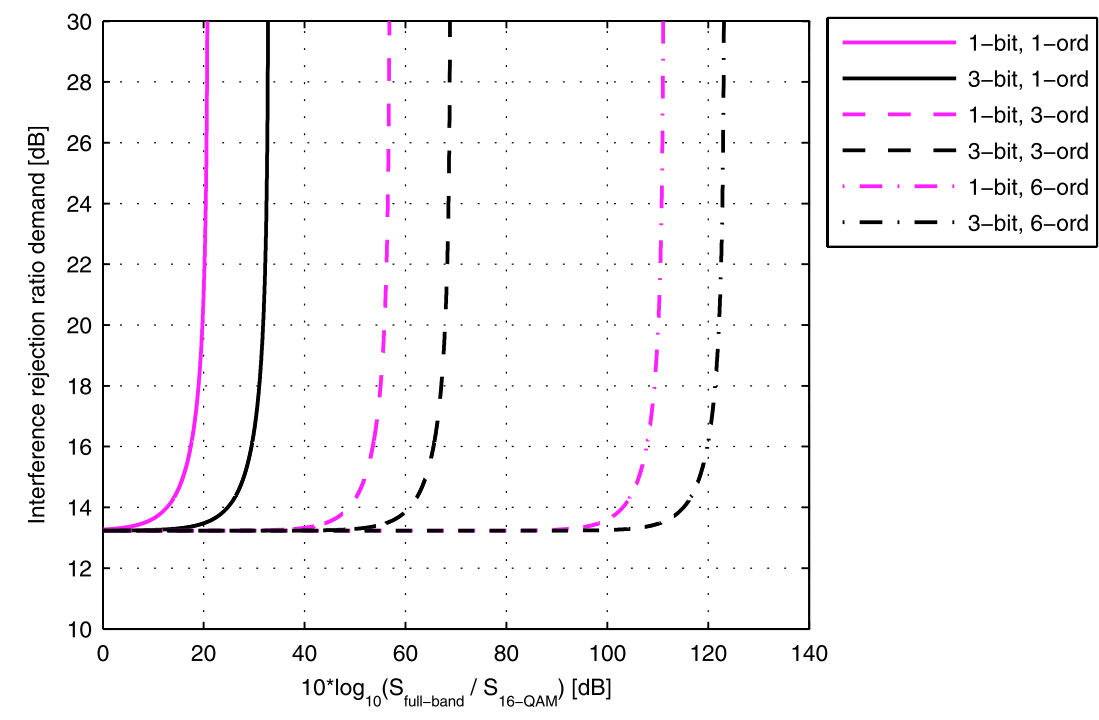

Figure 7 Demanded interference rejection ratio with different $\mathrm{Q} \Sigma \Delta \mathrm{M}$ setups as a function full-band signal power relative to the preferred signal power. SNRPRE at the ADC input and SINR target for detection are assumed 13 and $10 \mathrm{~dB}$, respectively, giving implementation margin of $3 \mathrm{~dB}$. 


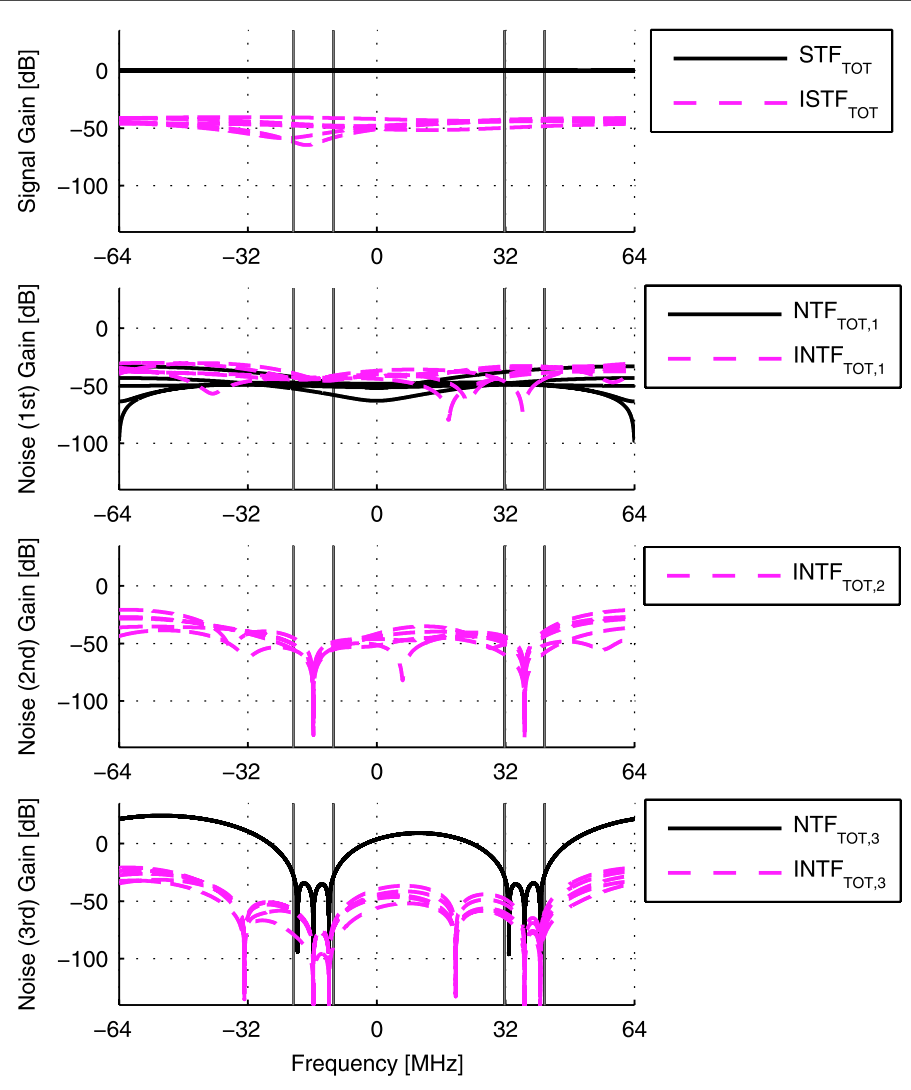

Figure 8 Three-stage Q $\Sigma \Delta M$ STF and ISTF (top) together with NTF and INTF for first-, second-, and third-stage quantization noises. Five independent random realizations in real gain values of feedback branches of both stages and flat STF design in all the stages. Multi-band reception of two information signals with center frequencies of 36.74 and $-15.74 \mathrm{MHz}$ is assumed. These bands are marked with gray solid lines in the plots.

$f_{\mathrm{C}, 1}=36.74 \mathrm{MHz}$ and $f_{\mathrm{C}, 2}=-15.74 \mathrm{MHz}$ is assumed with sampling frequency of $f_{\mathrm{S}}=128 \mathrm{MHz}$ (giving $T_{\mathrm{S}}=1 / f_{\mathrm{S}}=$ $7.8125 \mathrm{~ns})$. These bands, with bandwidth of $W_{1}=W_{2}=$ $10 \mathrm{MHz}$, are marked in Figures 8 and 9 with vertical gray lines. The frequency offsets from the center frequencies for the outermost NTF zeros are $f_{\text {offset }, 1}=f_{\text {offset }, 2}=\sqrt{3 / 20}$ * $10 \mathrm{MHz}=3.87 \mathrm{MHz}$, setting those zeros close to the interesting band edges.

The transfer functions of the stages are designed in the following manner, based on the above-described scenario and the discussion on design flow in Section 4. Third-order noise shaping is designed for both the signal bands, allowed by the overall NTF order of six. The first-stage NTF has unit-circle zeros on the center frequencies of the two signals, thus $\varphi_{\mathrm{NTF}, 1}^{(1)}=e^{j 2 \pi f_{\mathrm{C}, 1} T_{\mathrm{S}}}=e^{j 2 \pi 0.287}$ and $\varphi_{\mathrm{NTF}, 2}^{(1)}=e^{j 2 \pi f_{\mathrm{C}, 2} T_{\mathrm{S}}}=e^{-j 2 \pi 0.123}$. The second-stage zeros, $\varphi_{\mathrm{NTF}, 1}^{(2)}=e^{j 2 \pi\left(f_{\mathrm{C}, 1}+f_{\mathrm{offse}, 1}\right) T_{\mathrm{S}}}=e^{j 2 \pi 0.317}$ and $\varphi_{\mathrm{NTF}, 2}^{(2)}=$ $e^{j 2 \pi\left(f_{\mathrm{C}, 2}+f_{\text {offset }, 2}\right) T_{\mathrm{S}}}=e^{-j 2 \pi 0.093}$, are used to widen the noiseshaping notches toward higher frequencies. The lower frequencies of the interesting bands are notched by the third-stage NTF zeros $\varphi_{\mathrm{NTF}, 1}^{(3)}=e^{j 2 \pi\left(f_{\mathrm{C}, 1}-f_{\mathrm{offse}, 1}\right) T_{\mathrm{S}}}=e^{j 2 \pi 0.257}$ and $\varphi_{\mathrm{NTF}, 2}^{(3)}=e^{j 2 \pi\left(f_{\mathrm{C}, 2}-f_{\mathrm{offset}, 2}\right) T_{\mathrm{S}}}=e^{-j 2 \pi 0.153}$. With frequencyflat STF designs, the STF zeros and the common poles are placed in the origin. In the mirror-frequency-rejecting STF design considered for the first stage, the zeros of the first-stage STF are placed on respective mirror frequencies, giving $\varphi_{\mathrm{STF}, 1}^{(1)}=e^{-j 2 \pi 0.287}$ and $\varphi_{\mathrm{STF}, 2}^{(1)}=e^{j 2 \pi 0.123}$. At the same time, the common poles of the first-stage transfer functions are placed on the signal center frequencies, i.e., $\psi_{\text {common, } 1}^{(1)}=0.5 e^{j 2 \pi 0.287}$ and $\psi_{\text {common, } 2}^{(1)}=0.5 e^{-j 2 \pi 0.123}$, to highlight the STF selectivity and to maintain efficient noise shaping. Based on this design, the modulator coefficients are solved separately for each second-order stage as discussed above (see (48)-(59)). The digital filters $H_{1}^{\mathrm{D}}[z], H_{2}^{\mathrm{D}}[z]$, and $H_{3}^{\mathrm{D}}[z]$ are assumed to be matched perfectly to the analog transfer functions as described above.

\subsection{Transfer function analysis}

The transfer functions are evaluated and analyzed with randomly deviated real gain values (on I and Q rails) to model implementation inaccuracies. The deviation values are drawn from uniform distribution with maximum of $\pm 1 \%$ relative to the ideal value. Thus, for example, one 


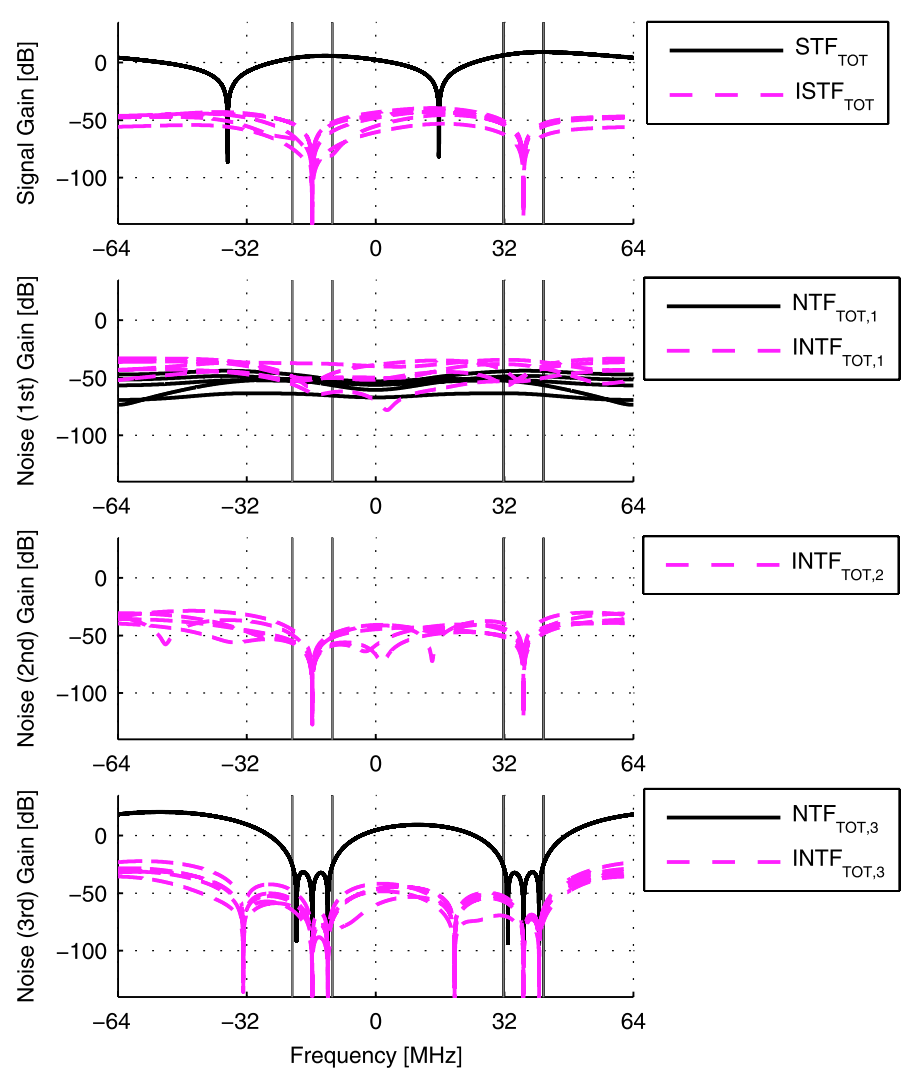

Figure 9 Three-stage Q $\Sigma \Delta M$ STF and ISTF (top) together with NTF and INTF for first-, second-, and third-stage quantization noises. Five independent random realizations in real gain values of feedback branches of both stages and mirror-frequency-rejecting STF design in first stage. Multi-band reception of two information signals with center frequencies of 36.74 and $-15.74 \mathrm{MHz}$ is assumed. These bands are marked with gray solid lines in the plots.

realization of the real part of the mismatched first-stage modulator feedback gain becomes $r_{\mathrm{re}, 1}^{(1)}=\left(1+\Delta_{r_{\mathrm{re}, 1}^{(1)}}\right) r_{\mathrm{re}}^{(1)}$, where $r_{\mathrm{re}, 1}^{(1)}$ is the implementation value and $r_{\mathrm{re}}^{(1)}$ the ideal value. First, the transfer functions are analyzed and illustrated in a case of second-order three-stage $\mathrm{Q} \Sigma \Delta \mathrm{M}$ with flat STF design in all the stages. The effects of I/Q imbalance are demonstrated by introducing mismatch to the feedback branches (coefficients $R^{(l)}$ and $S^{(l)}$ in Figure 5) of the stages. Five independent realizations of each transfer function, calculated with described mismatches, are plotted to demonstrate effects of inaccuracies on modulator response. The resulting transfer functions are shown in Figure 8. The overall ISTF response averages at $-50 \mathrm{~dB}$ level, varying between -40 and $-60 \mathrm{~dB}$ over the frequency. While the overall STF has $0 \mathrm{~dB}$ response, this results in averagely $50 \mathrm{~dB}$ image rejection for the input signal. The three latter plots in Figure 8 present the responses for the first-, second-, and third-stage quantization errors, respectively. The noise responses show that third-stage error is well shaped showing all six notches of the stages. Also the third-stage conjugate-noise (MFI stemming from the quantization error) is well attenuated, e.g., due to the digital filter $H_{3}^{\mathrm{D}}[z]=\mathrm{NTF}_{1}^{\mathrm{D}}[z] \mathrm{NTF}_{2}^{\mathrm{D}}[z] / \operatorname{STF}_{3}^{\mathrm{D}}[z]$, which gives nice attenuation on the interesting frequency bands. Firststage error is leaking to the output due to noncommutativity of mismatched complex transfer functions. However, attenuation on the preferred signal bands is still on average at the level of $-50 \mathrm{~dB}$ for the first-stage quantization error and $-40 \mathrm{~dB}$ for the conjugate component. However, when discussing noise responses, it should be remembered that large power variations as in the input blocker scenario are improbable. The second-stage nonconjugate noise is effectively canceled, but the conjugate version is visible at the output. This second-stage mirror-noise is, however, shaped by the NTF of the first stage, as mentioned in Section 4 , and thus nicely attenuated on the preferred signal bands.

Finally, in Figure 9, it is shown that mirror-frequencyrejecting STF design, proposed and discussed in Sections 3 and 4 , can effectively improve input image rejection in case of feedback branch mismatches also in a multi-stage modulator realizing multi-band conversion. This was shown in 


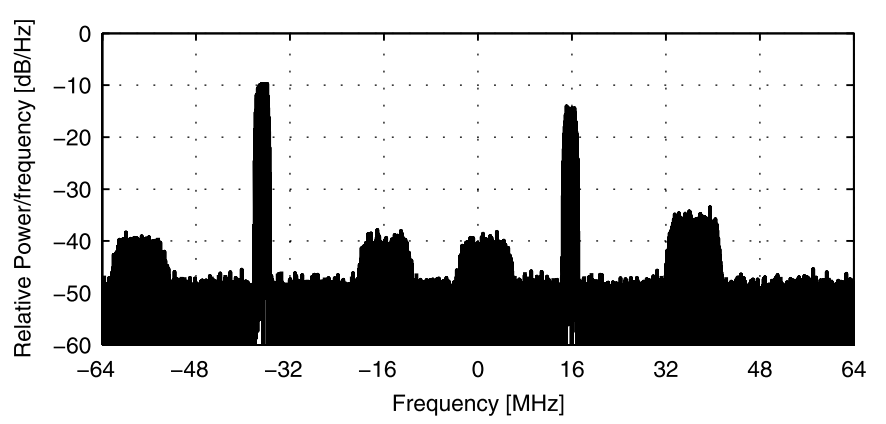

Figure 10 An example power spectral density of input signal used in the simulations. The preferred information signals are located around center frequencies of 36.74 and $-15.74 \mathrm{MHz}$ with 16-QAM and QPSK waveforms, respectively. Two strongest signals are located on the mirror frequencies of the preferred signals.

[19] and [30] for single-stage $\mathrm{Q} \Sigma \Delta \mathrm{Ms}$ and in [21] preliminarily for a two-stage modulator. Now, the closed-form analysis having arbitrary number of stages clearly confirms this. Specifically, in the three-stage example at hand, $20 \mathrm{~dB}$ average improvements in image rejection are seen over the information bands ( $-70 \mathrm{~dB}$ average ISTF response) compared to the frequency-flat STF. From Figure 9, it is seen that the ISTF notch is fairly narrow compared to the assumed bandwidth of the signal, stemming from the use of second-order $\mathrm{Q} \Sigma \Delta \mathrm{M}$ block which limits the number of the first-stage notches to two. However, MFI mitigation efficiency is more dependent on the bandwidth and power level of the blocking signal. For example, a narrow-band blocker at the mirror frequency of the preferred signal center frequency would be attenuated by over $80 \mathrm{~dB}$. In addition, it can be concluded, based on (7) and (33), that the characteristics of the third (or any subsequent) stage do not affect the processing of the original input signal or its image signal (conjugate response). On the other hand, increasing the order of the first stage would allow for more efficient STF design, resulting, e.g., in parallel notches in the ISTF at the interesting frequency band and thus improving the IRR even further.

\subsection{Computer simulations}

The conclusions of the transfer function analysis are confirmed herein with computer simulations and achievable interference rejection ratios are demonstrated. The multiband reception is simulated with an assumption of 16QAM and QPSK waveforms to be received on the center frequencies of $f_{\mathrm{C}, 1}=36.74 \mathrm{MHz}$ and $f_{\mathrm{C}, 2}=-15.74 \mathrm{MHz}$, respectively. Raised-cosine filters with roll-off of 0.25 are used for the pulse shaping, which together with symbol rate of $8 \mathrm{MHz}$, gives $10-\mathrm{MHz}$ waveform bandwidth. The QPSK band is received at $5 \mathrm{~dB}$ lower power level compared to 16-QAM band. Together with these preferred information signals, the overall input consists of four additional waveforms, of which two are located on the mir- ror frequencies of the signals of interest acting as blocking signals. In addition, a thermal input noise floor is present, limiting the 16-QAM and QPSK input SNRs to 13 and $8 \mathrm{~dB}$, respectively. An example of input spectrum is shown in Figure 10 including mirror-frequency blockers with $+20 \mathrm{~dB}$ power level compared to the preferred signals. The noninteresting signals consist of band-filtered white Gaussian noise with bandwidths of $3 \mathrm{MHz}$ for the mirrorfrequency blockers and $10 \mathrm{MHz}$ for the other two. Interference rejection ratio results are simulated with varying power levels for the two blockers.

In the interference rejection ratio simulations, true quantizers are used inside the modulator loop for the I and $Q$ rails to confirm the validity of the analytic model derived with the additive noise assumption. For generality, cases with 1-, 3-, and 8-bit quantizers are simulated and compared. In addition, frequency-flat and mirrorfrequency-rejecting STF designs are simulated with $1 \%$ I/Q mismatches in the feedback branches of the stages (coefficients $R^{(l)}$ and $S^{(l)}$ in Figure 5). These correspond to the maximum deviations used in the analytic transfer function analysis in Section 6.1. The mismatches are assigned randomly for the real and imaginary parts of each of the complex-valued coefficients, i.e., the real I-rail coefficients can be $1 \%$ smaller or larger than the ideal values, and the corresponding real Q-rail coefficients are deviated in the opposite direction. Thus, two examples (presenting the real part of the complex-valued $R^{(1)}$ ) of possible mismatched values of I-rail coefficients are $r_{\mathrm{re}, 1}^{(1)}=(1+0.01) r_{\mathrm{re}}^{(1)}$ and $r_{\mathrm{re}, 1}^{(1)}=(1-0.01) r_{\mathrm{re}}^{(1)}$. In these cases, the respective Q-rail real coefficient values are $r_{\mathrm{re}, 2}^{(1)}=(1-0.01) r_{\mathrm{re}}^{(1)}$ and $r_{\mathrm{re}, 2}^{(1)}=(1+0.01) r_{\mathrm{re}}^{(1)}$. The mismatches in each of the complex coefficients are independent of each other. The interference rejection ratio $\Gamma$ values are averaged over 25 independent random realizations of the mismatches. A single realization has input signal length of $2^{19}$ samples. The interference rejection ratio values are evaluated by subtract- 

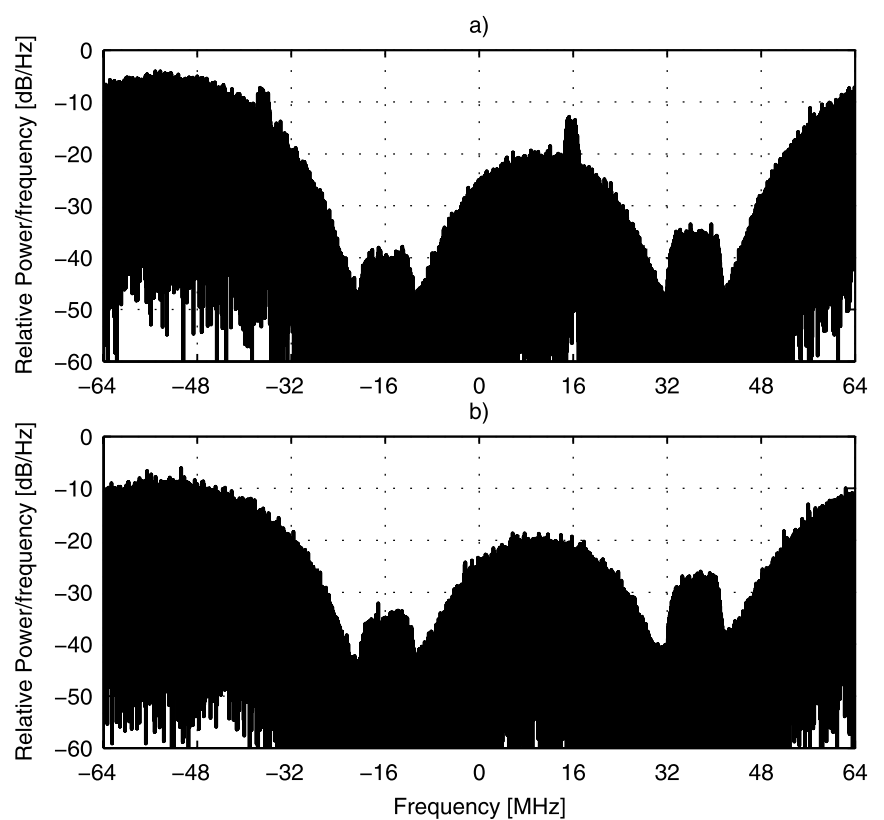

Figure 11 Example power spectral densities of output signals used in simulations: (a) with frequency-flat STF design, (b) with mirror-frequency-rejecting STF design. The preferred information signals are located around center frequencies of 36.74 and $-15.74 \mathrm{MHz}$ with 16-QAM and QPSK waveforms, respectively. 3-bit quantizers are used in all the three stages.

ing the output of an ideal $\mathrm{Q} \Sigma \Delta \mathrm{M}$ from the output of a mismatched $\mathrm{Q} \Sigma \Delta \mathrm{M}$, obtaining thus an estimate of distortion component. The interference rejection ratio itself is given as a ratio of the ideal output power on the preferred signal band and the distortion power estimate on the same band (see (38)-(41)). The presented power spectral densities are calculated with FFT-length of $2^{19}$ samples. The amplitudes of the real and imaginary parts of the overall received input signal are limited by the receiver automatic gain control mechanism to be equal to or less than 0.7 to avoid quantizer clipping (quantizer full scale range from -1 to 1 ), i.e., $\left|u_{\mathrm{I}, 1}(k)\right| \leq 0.7$ and $\left|u_{\mathrm{Q}, 1}(k)\right| \leq 0.7$ for all $k$. This limitation is maintained also when increasing the blocking signal power levels, which means that with increasing blocker input power, the useful signals are scaling down and become more and more sensitive to, e.g., quantization noise. For the sake of clarity, the output power spectral densities of the $\mathrm{Q} \Sigma \Delta \mathrm{M}$ are illustrated with frequency-flat and mirror-frequency-rejecting STF designs in Figure 11. From the plot (b), it is visible that, with the mirror-frequencyrejecting STF, the blockers around -36 and $16 \mathrm{MHz}$ are filtered out, and the preferred signals are more clearly above the noise compared to the case with flat STF design in the plot (a), thus indicating improved SINR. Further, Figure 12 shows the output power spectral densities of the two transfer function designs when 16-QAM waveform is disabled. Thus, it is possible to see the difference at the emerging MFI, originating from the blocker. In this scenario, the power spectral density of the frequency-flat STF design case shows interference peak on the assumed preferred signal band and mirror-frequency-rejecting STF design is able to push the MFI component below the noise floor.

Next, Figure 13 illustrates the interference rejection ratio results with 1-bit quantizers applied in the stages of a three-stage multi-band $\mathrm{Q} \Sigma \Delta \mathrm{M}$. The interference rejection ratios are calculated separately for the two received signals, separated with colors in the figures. In addition, frequency-flat and mirror-frequency-rejecting STF designs are compared. From Figure 13, it is clear that mirrorfrequency-rejecting STF design improves the interference rejection ratio of both the signals. The gain given by the STF design remains at $6 \mathrm{~dB}$ for the 16-QAM signal until relative blocker powers of $+20 \mathrm{~dB}$. For the QPSK signal, the corresponding gain is around $3 \mathrm{~dB}$. However, with the highest simulated blocking signal powers $(+40$ to $60 \mathrm{~dB}$ compared to the QPSK signal) the interference rejection ratio floors at the same level, independent of the STF design. These limited gain values of the mirror-frequencyrejecting STF design and similar flooring level between the designs originate from the distortion components other than the complex conjugate of the input signal. Thus, the signal quality is decreasing despite the input signal originating MFI being mitigated. For example, the leakage of the first-stage quantization error, already discussed in Section 6.1 , has a considerable role with 1-bit quantization, 


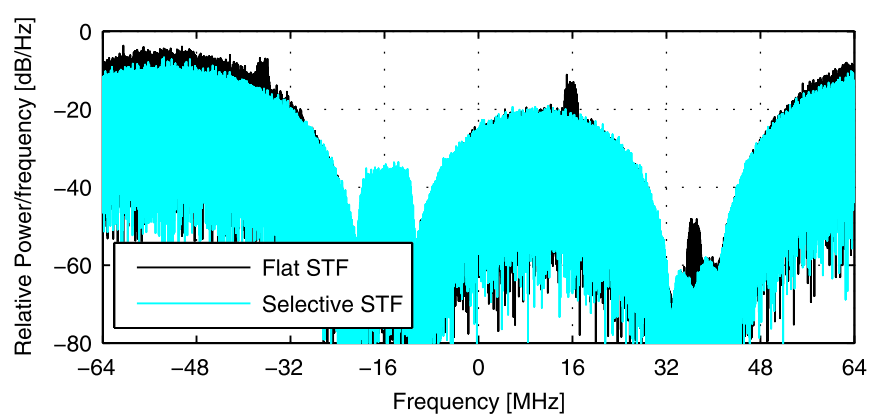

Figure 12 Example power spectral densities of output signals used in simulations with frequency-flat and mirror-frequency-rejecting STF designs with 16-QAM information signal around center frequency of $36.74 \mathrm{MHz}$ disabled to highlight image rejection properties. 3-bit quantizers are used in all the three stages.

nonshaped quantization error having significant power on the preferred signal bands. With increasing blocking signal powers, also the level of first-stage quantization error is increasing compared to the preferred signals, and this decreases the interference rejection ratio values regardless of the STF design.

Overall, the achievable interference rejection ratios are well in line with demands derived in Section 5. From 16QAM signal point of view, the demanded rejection (see Figure 7) is fulfilled with selective STF up to the relative blocker power of $20 \mathrm{~dB}$. At this point, the full-band power to the 16-QAM signal power ratio can be approximated to be $23 \mathrm{~dB}$, neglecting the minor effect of other out-of-band signals than the two mirror-frequency blockers. At this point, the achieved interference rejection ratio of $15 \mathrm{~dB}$ fulfills the demand of $13 \mathrm{~dB}$ with 3-bit quantization (see Figure 7).

The results with 3-bit quantizers, given in Figure 14, support the above conclusions. When the levels of the error components are decreased due to additional quantization bits, the gain given by the mirror-frequency-rejecting STF design is more pronounced. The gain increases when the blocking signal power cross the $0 \mathrm{~dB}$ level, due to the increasing amount of MFI stemming from the input signal. This gain remains around $15 \mathrm{~dB}$ for the $16-\mathrm{QAM}$ signal and $10 \mathrm{~dB}$ for the QPSK signal at the relative blocker power ranging from +10 to $+60 \mathrm{~dB}$. This is because of the decreased levels of the quantization error components. Especially in wideband $C R$ receivers operating in challenging radio conditions with strong out-of-band signal dynamics,

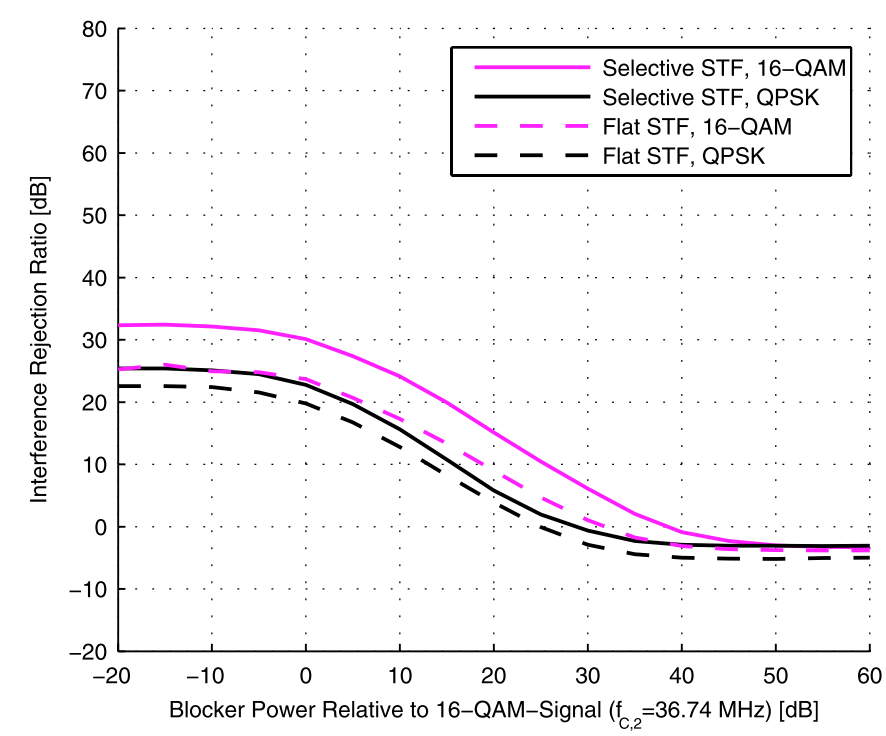

Figure 13 Interference rejection ratios for preferred signals with three-stage $Q \Sigma \Delta M$, using 1-bit quantizers at each stage, as a function of blocker signal power. Frequency-flat ("Flat STF") and mirror-frequency-rejecting STF ("Selective STF") designs are simulated. 


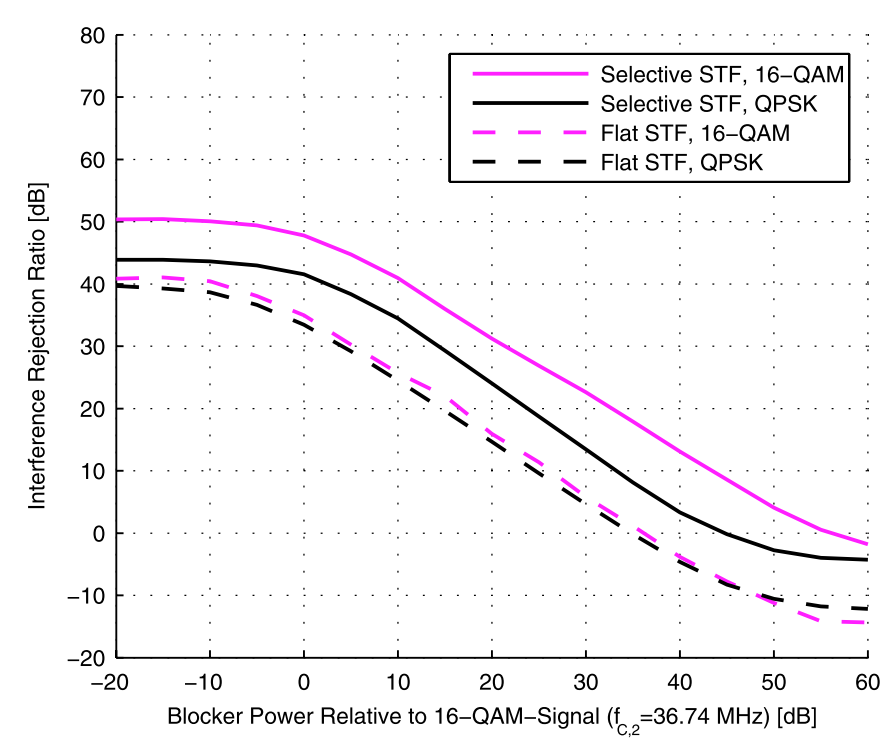

Figure 14 Interference rejection ratios for preferred signals with three-stage $Q \Sigma \Delta M$, using 3-bit quantizers at each stage, as a function of blocker signal power. Frequency-flat ("Flat STF") and mirror-frequency-rejecting STF ("Selective STF") designs are simulated.

the shown 10-15 $\mathrm{dB}$ gains are very valuable, improving the robustness of the receiver significantly.

Comparing these results to the set demand for the interference rejection ratio, it can be seen that 16-QAM with selective STF fulfills the demand up to the relative blocker levels of $+40 \mathrm{~dB}$. In this scenario, full-band power to the 16-QAM power ratio is approximately $43 \mathrm{~dB}$, which gives interference rejection ratio demand of $13 \mathrm{~dB}$ with 3-bit quantization (see Figure 7) matching to the $13 \mathrm{~dB}$ result seen in Figure 14.

Finally, Figure 15 provides the results with 8-bit quantizers used in the stages (mainly for reference, without interference rejection target). In this scenario, the quantization error levels are pushed even further down, and the MFI from the input remains as a dominant error source. The interference rejection ratio values in Figure 15 pronounce

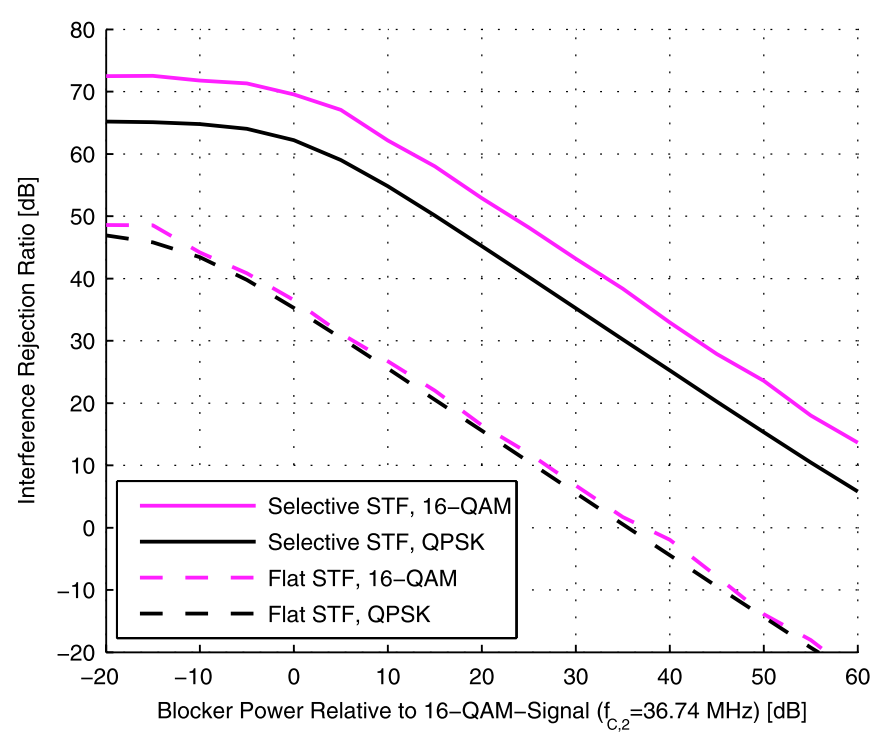

Figure 15 Interference rejection ratios for preferred signals with three-stage $Q \Sigma \Delta M$, using 8-bit quantizers at each stage, as a function of blocker signal power. Frequency-flat ("Flat STF") and mirror-frequency-rejecting STF ("Selective STF") designs are simulated. 
the efficiency of the mirror-frequency-rejecting STF design in mitigating this distortion. The gains achieved with this design remain at the levels of 40 and $30 \mathrm{~dB}$ for the 16-QAM and QPSK signals, respectively, with the relative blocker levels above $+10 \mathrm{~dB}$. Again, these findings support the capability of the mirror-frequency STF design in input signal-originating MFI mitigation. However, with limited quantization precisions (such as the 1-bit case), the role of the other distortion sources is also significant.

\section{Conclusions}

This article provided an analytic transfer function model for I/Q imbalance effects in a second-order multistage $\mathrm{Q} \Sigma \Delta \mathrm{M}$ with arbitrary number of stages. For each of the stages, input branches, loop filters, and feedback branches were modeled as potential mismatch sources. Mirrorfrequency-rejecting STF design was proposed for the first stage of multistage $\mathrm{Q} \Sigma \Delta \mathrm{Ms}$ as an efficient tool against MFI due to feedback mismatches. Thereafter, based on the derived model, it was concluded that in three-stage $\mathrm{Q} \Sigma \Delta \mathrm{M}$ the mirror-frequency-rejecting STF design in the first stage was able to improve the image rejection of the modulator by $20 \mathrm{~dB}$, when feedback branch I/Q mismatches were considered. This technique improves the image rejection of a multistage $\mathrm{Q} \Sigma \Delta \mathrm{M}$ without any additional electronics. The MFI mitigation capability of the mirror-frequency-rejecting STF design was also confirmed with computer simulation-based interference rejection ratio calculations. Based on the simulations, it was concluded that this STF design is able to significantly reduce the MFI on the preferred signal bands. However, with limited quantization precision, the quantization error-based additional distortion components restrict the achievable interference rejection ratio.

In general, multi-band design aimed toward CR receivers was discussed, and the three-stage $\mathrm{Q} \Sigma \Delta \mathrm{M}$ was found to offer valuable degrees of freedom in transfer function design to receive scattered frequency slices efficiently. This multi-band reception scheme is a promising possibility for frequency agile A/D conversion for CR. The transfer functions of a multistage $\mathrm{Q} \Sigma \Delta \mathrm{M}$ can be reconfigured straightforwardly based on the spectrum-sensing information. This was shown with parameterization of the zeros and the poles of the stage NTFs and STFs. The proposed design principles and flow can be realized with information about the center frequencies and the bandwidths of the signals to be received.

While the mirror-frequency-rejecting STF design was shown to be effective against input blocker mirroring, the closed-form analysis also showed that first-stage quantization noise leakage due to noncommutativity of the complex transfer functions under I/Q imbalance is a problem in multistage $\mathrm{Q} \Sigma \Delta$ Ms. This problem was confronted also in interference rejection ratio simulations. Developing ways to mitigate the noise leakage would increase the resolution of the ADC and increase the role of the proposed mirror-frequency-rejecting STF design even further. This will be addressed in future research.

\section{Acknowledgement}

This study was supported by the Academy of Finland (under the project entitled "Digitally-enhanced RF for cognitive radio devices"), the Finnish Funding Agency for Technology and Innovation (Tekes, under the projects entitled "Advanced techniques for RF impairment mitigation in future wireless radio systems" and "Enabling methods for dynamic spectrum access and cognitive radio"), Austrian Center of Competence in Mechatronics (ACCM, under the project entitled "Wireless communication technologies"), Tampere University of Technology Graduate School, HPY Research Foundation, and Nokia Foundation.

\section{Competing interests}

The authors declare that they have no competing interests.

Received: 30 April 2011 Accepted: 12 October 2011 Published: 12 October 2011

\section{References}

1. IF Akyildiz, W-Y Lee, MC Vuran, S Mohanty, NeXt generation/dynamic spectrum access/cognitive radio wireless networks: a survey. Comput. Networks 50, 2127-2159 (2006)

2. M Mueck, A Piipponen, K Kalliojärvi, G Dimitrakopoulos, KTsagkaris, P Demestichas, F Casadevall, J Perez-Romero, O Sallent, G Baldini, S Filin, H Harada, M Debbah, T Haustein, J Gebert, B Deschamps, P Bender, M Street, S Kandeepan, I Lota, A Hayar, ETSI reconfigurable radio systems: status and future directions on software defined radio and cognitive radio standards. IEEE Commun. Mag. 48, 78-86 (2010)

3. P-I Mak, S-P U, RP Martins, Transceiver architecture selection: review, state-of-the-art survey and case study. IEEE Circuits Syst. Mag. 7, 6-25 (2007)

4. R Bagheri, A Mirzaei, ME Heidari, S Chehrazi, M Lee, M Mikhemar, WK Tang, AA Abidi, Software-defined radio receiver: dream to reality. IEEE Commun. Mag. 44, 111-118 (2006)

5. Z Ru, NA Moseley, E Klumperink, B Nauta, Digitally enhanced software-defined radio receiver robust to out-of-band interference. IEEE J. Solid-State Circuits 44, 3359-3375 (2009)

6. B Razavi, Cognitive radio design challenges and techniques. IEEE J. Solid-State Circuits 45, 1542-1553 (2010)

7. PB Kenington, L Astier, Power consumption of A/D converters for software radio applications. IEEE Trans. Veh. Technol. 49, 643-650 (2000)

8. M Ismail, D Rodríguez de Llera González (eds.), Radio Design in Nano-meter Technologies (Springer, Dordrecht, 2006)

9. J Yang, RW Brodersen, D Tse, Addressing the dynamic range problem in cognitive radios, in Proceedings of the IEEE International Conference on Communications, Glasgow, Scotland, 24-28 June 2007

10. AA Abidi, The path to the software-defined radio receiver. IEEE J. Solid-State Circuits 42, 954-966 (2007)

11. B Le, TW Rondeau, JH Reed, CW Bostian, Analog-to-digital converters. IEEE Signal Process. Mag. 22, 69-77 (2005)

12. N Vun, AB Premkumar, ADC systems for SDR digital front-end, in Proceedings of the 9th International Symposium on Consumer Electronics, Macau, Hong Kong, 14-16 June 2005

13. PA Aziz, HV Sorensen, J van der Spiegel, An overview of sigma-delta converters: how a 1-bit ADC achieves more than 16-bit resolution. IEEE Signal Process. Mag. 13, 61-84 (1996)

14. A Rusu, B Dong, M Ismail, Putting the "flex" in flexible mobile wireless radios. IEEE Circuits Devices Mag. 22, 24-30 (2006)

15. A Silva, J Guilherme, N Horta, Reconfigurable multi-mode sigma-delta modulator for 4G mobile terminals. Integration. The VLSI J. 42, 34-46 (2009)

16. J Crols, MSJ Steyaert, Low-IF topologies for high-performance analog front ends of fully integrated receivers. IEEE Trans. Circuits Syst. II Analog Digit. Signal Process. 45, 269-282 (1998) 
17. N Jouida, C Rebai, G Ghazel, D Dallet, Comparative study between continuous-time real and quadrature bandpass delta sigma modulator for mul-tistandard radio receiver, in Proceedings of the Instrumentation Measurement Technology Conference, Warsaw, Poland, 1-3 May 2007

18. S Jantzi, K Martin, A Sedra, A quadrature bandpass delta-sigma modulator for digital radio. IEEE J. Solid-State Circuits. 32, 1935-1950 (1997)

19. J Marttila, M Allén, M Valkama, Quadrature $\Sigma \Delta$ modulation for cognitive radio - I/Q imbalance analysis and complex multiband principle. Circuits, Syst. and Signal Process. 30, 775-797, (2011)

20. DD Ariananda, MK Lakshmanan, H Nikoo, A survey on spectrum sensing techniques for cognitive radio, in Proceedings of the 2 nd International Workshop on Cognitive Radio and Advanced Spectrum Management Aalborg, Denmark, 18-20 May 2009

21. J Marttila, M Allén, M Valkama, Design and analysis of multi-stage quadrature sigma-delta A/D converter for cognitive radio receivers, in Proceedings of the 16th IEEE International Workshop on Computer-Aided Modeling Analysis and Design of Communication Links and Networks, Kyoto, Japan, 10-11 June 2011

22. YBN Kumar, STalay, F Maloberti, Complex cascaded bandpass $\Sigma \Delta$ ADC design, in Proceedings of the IEEE International Symposium on Circuits and Systems, Taipei, Taiwan, 24-27 May 2009

23. Y Tang, K-W Cheng, S Gupta, J Paramesh, DJ Allstot, Cascaded complex ADCs with adaptive digital calibration for I/Q mismatch. IEEE Trans. Circuits Syst. I Reg. Papers 55, 817-827 (2008)

24. M Valkama, J Pirskanen, M Renfors, Signal processing challenges for applying software radio principles in future wireless terminals: an overview. Int. J. Commun. Syst. 15, 741-769 (2002)

25. S Jantzi, KW Martin, AS Sedra, The effects of mismatch in complex bandpass $\Delta \Sigma$ modulators, in Proceedings of the IEEE International Symposium on Circuits and Systems, Atlanta, GA, 12-15 May 1996

26. LJ Breems, EC Dijkmans, JH Huijsing, A quadrature data-dependent DEM algorithm to improve image rejection of a complex modulator. IEEE J. Solid-State Circuits 36, 1879-1886 (2001)

27. L Yu, WM Snelgrove, A novel adaptive mismatch cancellation system for quadrature IF radio receivers. IEEE Trans. Circuits Syst. II Analog Digit. Signal Process. 46, 789-801 (1999)

28. K-P Pun, C-S Choy, C-F Chan, JE da Franca, An I/Q mismatch-free switched-capacitor complex sigma-delta modulator. IEEE Trans. Circuits Syst. I Express Briefs 51, 254-256 (2004)

29. S Reekmans, P Rombouts, L Weyten, Mismatch insensitive double-sampling quadrature bandpass $\Sigma \Delta$ modulation. IEEE Trans. Circuits Syst. I Regular Papers 54, 2599-2607 (2007)

30. J Marttila, M Allén, M Valkama, Response analysis and design of second-order quadrature $\Sigma \Delta$ modulators with applications in cognitive radio devices, in 54th IEEE International Midwest Symposium on Circuits and Systems, Seoul, South-Korea, 7-10 August 2011 (invited paper)

31. N Jouida, C Rebai, G Ghazel, Built-in filtering for out-of-channel interferers in continuous-time quadrature bandpass delta sigma modulators, in Proceedings of the 14th IEEE International Conference on Electronics, Circuits Systems, Marrakech, Morocco, 11-14 December 2007

32. K Hyungseok, L Junghan, T Copani, S Bazarjani, S Kiaei, B Bakkaloglu, Adaptive blocker rejection continuous-time $\Sigma \Delta$ ADC for mobile WiMAX applications. IEEE J. Solid-State Circuits 44, 2766-2779 (2009)

33. R Schreier, GC Temes, Understanding Delta-Sigma Data Converters (Wiley, Hoboken, 2005)

34. P Kiss, Adaptive digital compensation of analog circuit imperfections for cascaded delta-sigma analog-to-digital converters, Dissertation, "Politehnica" Univ. Timisoara, Romania, 1999

35. G Cauwenberghs, GC Temes, Adaptive digital correction of analog errors in MASH ADCs. I. Off-line and blind on-line calibration. IEEE Trans. Circuits Syst. II Analog Digit. Signal Process. 47, 621-628 (2000)

36. R Schreier, An empirical study of high-order single-bit delta-sigma modulators. IEEE Trans. Circuits Syst. II Analog Digit. Signal Process. 40, 461-466 (1993)

doi:10.1186/1687-1499-2011-130

Cite this article as: Marttila et al: Multistage quadrature sigma-delta modulators for reconfigurable multi-band analog-digital interface in cognitive radio devices. EURASIP Journal on Wireless Communications and Networking 2011 2011:130.

\section{Submit your manuscript to a SpringerOpen ${ }^{\circ}$ journal and benefit from:}

- Convenient online submission

- Rigorous peer review

- Immediate publication on acceptance

- Open access: articles freely available online

- High visibility within the field

- Retaining the copyright to your article

Submit your next manuscript at $\boldsymbol{\triangleright}$ springeropen.com 